\title{
GAP OPENING IN THE ESSENTIAL SPECTRUM OF THE ELASTICITY THEORY PROBLEM IN A PERIODIC HALF-LAYER
}

\author{
S. A. NAZAROV
}

\begin{abstract}
Rayleigh waves are studied in an elastic half-layer with a periodic end and rigidly clamped faces. It is established that the essential spectrum of the corresponding problem of elasticity theory has a band structure, and an example of a waveguide is presented in which a gap opens in the essential spectrum; i.e., an interval arises that contains points of an at most discrete spectrum.
\end{abstract}

\section{$\S 1$. INTRODUCTION}

1. Preamble. In the case of a homogeneous isotropic elastic half-space, surface waves were discovered by Lord Rayleigh 1, and since then many investigations devoted to similar effects have appeared (see a survey of modern literature in [2, and also the paper [3], which is absent in [2]). A Rayleigh wave is a plane wave of the form

$$
\exp \left(i k x_{2}\right) U\left(x_{3}\right), \quad x=\left(x_{1}, x_{2}, x_{3}\right) \in \mathbb{R}_{-}^{3}, \quad k \in \mathbb{R},
$$

with a vector-valued factor $U(z)$ decaying exponentially as $z=x_{3} \rightarrow-\infty$. The arising of such waves explains specific wave processes in elastic bodies.

The wave number $k \in \overline{\mathbb{R}_{+}}=[0,+\infty)$ determines a frequency cutoff $\omega_{\dagger}(k)$ above which, i.e., for $\omega \geq \omega_{\dagger}(k)$, the wave (1.1) exists necessarily. In the present paper we deal with a problem related to a similar phenomenon. Namely, we study an elastic, but not necessarily homogeneous and isotropic cushion $\Omega_{0}$ having the form of a half-layer with a periodic end and rigidly clamped side faces (see Figure 1, where the clamped surface is shadowed). Some Rayleigh waves decaying exponentially as $z \rightarrow-\infty$ can propagate along the end of the cushion, and if $\Omega_{0}$ is a cylinder $\Delta \times \mathbb{R}$, then we have a single cutoff $\omega_{\dagger}>0$; i.e., the corresponding operator of the elasticity theory system acquires a continuous spectrum $\left[\omega_{\dagger},+\infty\right)$. Our main goal in this paper is to show that, in the periodic case, a gap can open in the essential spectrum; i.e., an interval can exist the ends of which belong to the continuous spectrum, but inside which only points of the discrete spectrum may occur. Some of the results were announced earlier in [4.

2. Setting of the problem. Let $\Pi$ be a domain in Euclidean space $\mathbb{R}^{3}$ (see Figures 2-4; Figure 4 shows a section of the 3-dimensional cell depicted in Figure 3). It is assumed that $\Pi(-H) \subset \Pi \subset \Pi(H)$, where

$$
\Pi(H)=\left\{x=(y, z): y=\left(y_{1}, y_{2}\right),\left|y_{p}\right|<l_{p} / 2, p=1,2, z<H\right\},
$$

2000 Mathematics Subject Classification. Primary 35Q72.

Key words and phrases. Rayleigh waves, essential spectrum, band structure.

Supported by the Netherlands Organization for Scientific Research (NWO) and RFBR, joint project 047.017.020. 


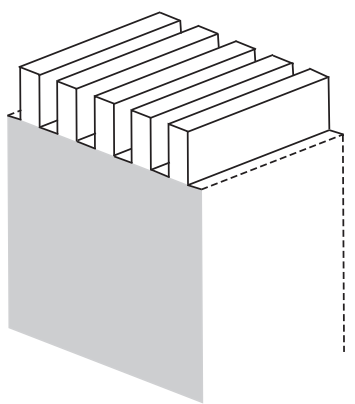

Figure 1. A periodic half-layer.

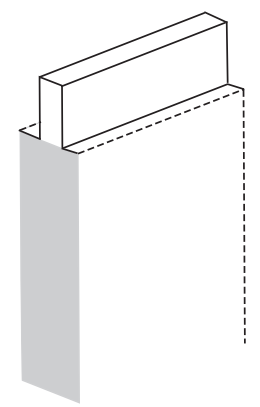

FigURE 2. A periodicity cell.

and $l_{p}$ and $H$ are positive quantities. By scaling, we reduce the size $l_{1}$ to unity; after that, we put $l=l_{2}$. A half-layer $\Xi$ with a periodic structure will be introduced as the interior of the set

$$
\bar{\Omega}_{0}=\bigcup_{j \in \mathbb{Z}} \bar{\Pi}_{j},
$$

where $\mathbb{Z}=\{0, \pm 1, \ldots\}$ and $\Pi_{j}=\left\{x:\left(y_{1}-j, y, z\right) \in \Pi\right\}$. We assume that $\Omega_{0}$ is a domain with Lipschitz boundary and, in particular, a connected set (see Figures 1 and 5, which correspond to the cells in Figures 2 and 4). Finally, let $\Omega$ be yet another Lipschitz domain that coincides with $\Omega_{0}$ off a ball $\mathbb{B}_{R^{0}}=\left\{x:|x|<R^{0}\right\}$ with a large radius $R^{0}>0$ (cf. Figures 5 and 6 ). Viewing the domain $\Omega$ as a solid, we consider the spectral problem of elasticity theory in a matrix form (see [5, 6]):

$$
\begin{aligned}
D\left(-\nabla_{x}\right)^{\top} A(x) D\left(\nabla_{x}\right) u(x) & =\lambda \rho(x) u(x), & & x \in \Omega, \\
D(\nu(x))^{\top} A(x) D\left(\nabla_{x}\right) u(x) & =0, & & x \in \Sigma, \\
u(x) & =0, & & x \in \Gamma .
\end{aligned}
$$

We explain the notation adopted. The displacement vector is regarded as a column $\left(u_{1}, u_{2}, u_{3}\right)^{\top}$ in $\mathbb{R}^{3}$ (here $\top$ means transposition), and $u_{p}$ and $u_{3}$ are the projections of $u$ to the axes $x_{p}=y_{p}$ and $x_{3}=z$, respectively, $p=1,2$. The columns of strains and 


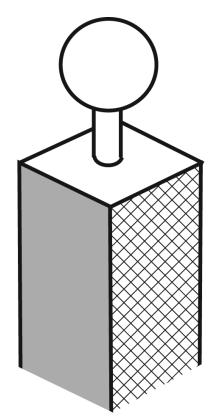

FiguRE 3. A mushroom-like periodicity cell.

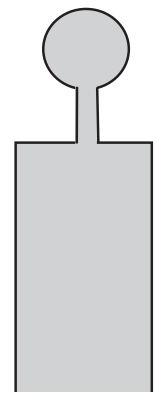

Figure 4. The two-dimensional cross-section of a cell.

stresses, $\varepsilon(u)$ and $\sigma(u)$, are defined respectively by the formulas

$$
\begin{aligned}
& \varepsilon(u)=\left(\varepsilon_{11}, \varepsilon_{22}, \varepsilon_{33}, \sqrt{2} \varepsilon_{23}, \sqrt{2} \varepsilon_{31}, \sqrt{2} \varepsilon_{12}\right)^{\top}, \\
& \sigma(u)=\left(\sigma_{11}, \sigma_{22}, \sigma_{33}, \sqrt{2} \sigma_{23}, \sqrt{2} \sigma_{31}, \sqrt{2} \sigma_{12}\right)^{\top}, \\
& \varepsilon(u ; x)=D\left(\nabla_{x}\right) u(x), \quad \sigma(u ; x)=A(x) \varepsilon(u ; x),
\end{aligned}
$$

where $\varepsilon_{j k}=\frac{1}{2}\left(\partial_{j} u_{k}+\partial_{k} u_{j}\right)$ and the $\sigma_{j k}$ are the Cartesian coordinates of the strain and stress tensors (the factors $\sqrt{2}$ are involved in the definition (1.7) for equating the natural norms of the tensors and the corresponding columns; see [6, $\S 2.1]$ ), and $D\left(\nabla_{x}\right)$ is the following $(6 \times 3)$-matrix of differential operators:

$$
\begin{aligned}
D\left(\nabla_{x}\right)^{\top} & =\left(\begin{array}{cccccc}
\partial_{1} & 0 & 0 & 0 & 2^{-1 / 2} \partial_{3} & 2^{-1 / 2} \partial_{2} \\
0 & \partial_{2} & 0 & 2^{-1 / 2} \partial_{3} & 0 & 2^{-1 / 2} \partial_{1} \\
0 & 0 & \partial_{3} & 2^{-1 / 2} \partial_{2} & 2^{-1 / 2} \partial_{1} & 0
\end{array}\right), \\
\nabla_{x} & =\left(\begin{array}{c}
\partial_{1} \\
\partial_{2} \\
\partial_{3}
\end{array}\right), \quad \partial_{j}=\frac{\partial}{\partial x_{j}} .
\end{aligned}
$$

The material density $\rho$ and the elements $A_{p q}$ of the $(6 \times 6)$-matrix $A$ of elastic modules are bounded measurable functions on $\Omega$ that are subject to the positivity condition

$$
c_{A}|\xi|^{2} \leq \xi^{\top} A(x) \xi \leq C_{A}|\xi|^{2}, \quad \xi \in \mathbb{R}^{6}, \quad c_{\rho} \leq \rho(x) \leq C_{\rho},
$$

and the stabilization condition

$$
\left|A_{p q}(x)-A_{p q}^{0}\right| \leq c_{0} \exp \left(-\delta_{0}|x|\right), \quad\left|\rho(x)-\rho_{0}\right| \leq c_{0} \exp \left(-\delta_{0}|x|\right),
$$




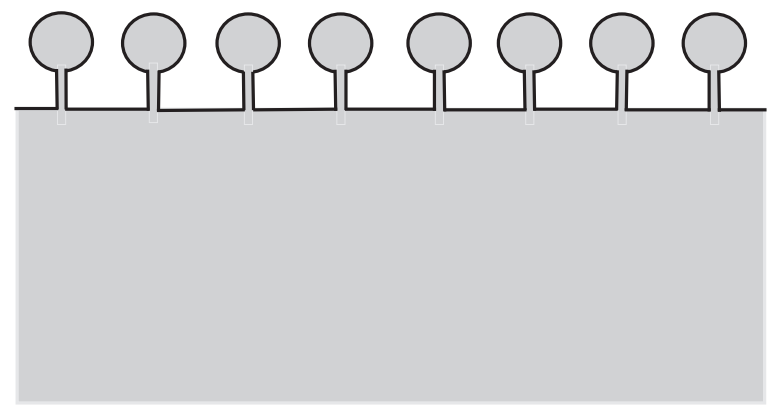

Figure 5. The cross section of a periodic half-layer.

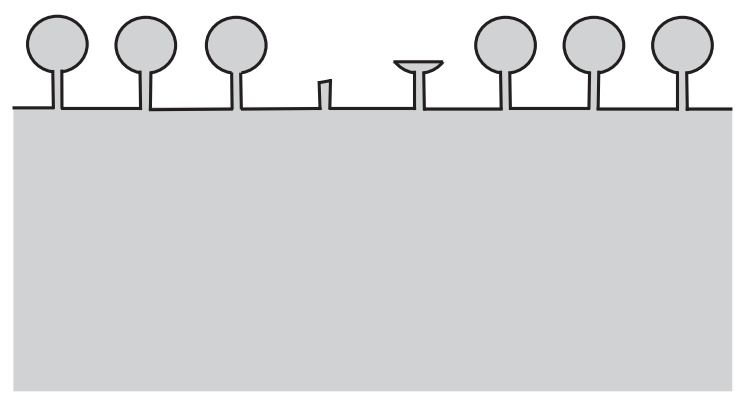

FiguRE 6 . The cross section of a periodic half-layer perturbed locally.

where $c_{A}, C_{A}, c_{\rho}, C_{\rho}$, and $c_{0}, \delta_{0}$ are positive numbers, and relations (1.9) remain valid for the numerical $(6 \times 6)$-matrix $A^{0}$ and the scalar $\rho_{0}$ in formula (1.10), probably, with new positive constants $c_{A}^{0}, C_{A}^{0}, c_{\rho}^{0}, C_{\rho}^{0}$. The spectral parameter $\lambda$ is the squared frequency of elastic oscillations. The Dirichlet boundary conditions (1.6) correspond to the rigid clamping of the surface $\Gamma$ which, by assumption, includes the half-planes $\left\{x: y_{2}= \pm l\right.$, $z<-H\}$, while the Neumann condition, involving the outward unit normal, means that the other part $\Sigma=\partial \Omega \backslash \bar{\Gamma}$ of the surface is traction-free. In the sequel, we shall need the following restriction: off the ball $\mathbb{B}_{R}$, the surface $\Gamma$ is invariant under shifts by \pm 1 along the $x_{1}$-axis (see Figure 7 , where the surface $\Gamma$ (the shadowed domain) and the ball $\mathbb{B}_{R}$ are depicted schematically). Formally, the requirements listed look like this:

$$
\begin{aligned}
\Gamma^{ \pm} & =\left\{x \in \Gamma: \pm y_{2} \geq 0\right\}, \quad \Gamma^{ \pm} \backslash \mathbb{B}_{R^{0}}=\Gamma_{0}^{ \pm} \backslash \mathbb{B}_{R^{0}}, \\
\Gamma_{0}^{ \pm} & =\left\{x:\left(y_{1} \pm 1, y_{2}, z\right) \in \Gamma_{0}^{ \pm}\right\}, \quad\left\{x: y_{1}= \pm 1 / 2, z<-H\right\} \subset \Gamma_{0}^{ \pm}, \\
\Gamma_{0} & =\Gamma_{0}^{+} \cup \Gamma_{0}^{-} .
\end{aligned}
$$

Elastic bodies with a periodic structure as described occur in practice; imposing the boundary conditions (1.5) and (1.6) allows us to interpret $\Omega$ as an elastic cushion clamped between two absolutely rigid stamps. Part of the cushion goes beyond the stamp's edges (in our notation these are the lines where the type of the boundary conditions changes). The discrete spectrum and the continuous spectrum of problem (1.4)-(1.6) are related to the arising of elastic trap modes and also waves that propagate along the edge and decay exponentially as the distance from the edge grows (see the paper [7] and the surveys [2, 8]).

Since the boundary of the body and the coefficients of the differential operators involved may happen to be irregular, we understand problem (1.4)-(1.6) as the following 


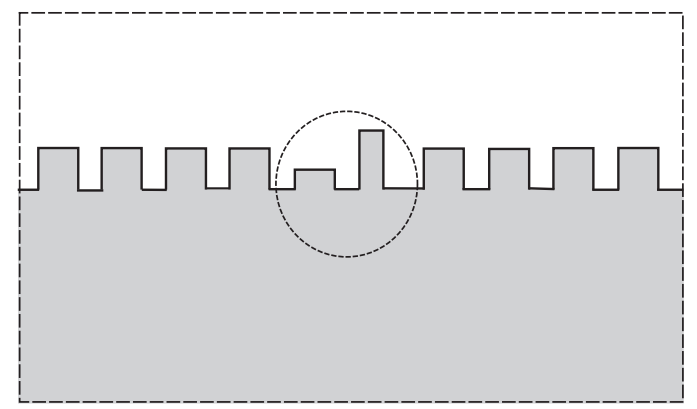

Figure 7. A periodic clamping zone with local perturbation.

integral identity [9, 10]:

$$
\left(A D\left(\nabla_{x}\right) u, D\left(\nabla_{x}\right) v\right)_{\Omega}=\lambda(\rho u, v)_{\Omega}, \quad v \in \stackrel{\circ}{H}^{1}(\Omega ; \Gamma)^{3} .
$$

Here $(,)_{\Omega}$ is the inner product in the Lebesgue space $L_{2}(\Omega), \stackrel{\circ}{H}^{1}(\Omega ; \Gamma)$ is the Sobolev space of functions that vanish on $\Gamma$ (the mark ${ }^{\circ}$ above $H$ ), and the upper index 3 indicates the number of vector components. We omit the size of the vectors in the notation for norms and inner products. In the terminology of [10], the Neumann condition (1.5) and the Dirichlet condition (1.6) are (respectively) the intrinsic condition and the stable condition for the system of equations of elasticity theory.

The Hilbert space $\stackrel{\circ}{H}^{1}(\Omega ; \Gamma)^{3}$ equipped with a specific inner product, namely

$$
\langle u, v\rangle=\left(A D\left(\nabla_{x}\right) u,\left(D\left(\nabla_{x}\right) v\right)_{\Omega}+(\rho u, v)_{\Omega}\right.
$$

(see the Korn inequalities (3.1) and (2.34) below), will be denoted by $\mathcal{H}$. Half of the quantity $\langle u, u\rangle$ is the sum of the elastic and kinetic energies. The formula

$$
\langle\mathcal{K} u, v\rangle=(\rho u, v)_{\Omega}, \quad u, v \in \mathcal{H},
$$

determines a positive, symmetric, and continuous (and hence, selfadjoint) operator $\mathcal{K}$ in the space $\mathcal{H}$. Since the first term on the right-hand side in (1.14) is nonnegative, the norm of $\mathcal{K}$ does not exceed 1. Therefore, Hilbert space operator theory (see, e.g., [11, $\S \S 3.7,10.2])$ guarantees that the spectrum of $\mathcal{K}$ lies on the interval $[0,1]$ of the real axis in the complex plane $\mathbb{C}$, and the set $\mathbb{C} \backslash[0,1]$ is included in the resolvent set of $\mathcal{K}$.

The definitions (1.13), (1.14) and the spectral parameter change

$$
\mu=(1+\lambda)^{-1}
$$

allow us to rewrite the variational problem (1.12) as an abstract spectral equation:

$$
\mathcal{K} u=\mu u \text {. }
$$

Due to (1.15), the $\lambda$-spectrum of problem (1.12) inherits all properties of the $\mu$-spectrum of equation (1.16) except those related to the point $\mu=0$, which corresponds to the infinitely remote $\lambda$. Therefore, in what follows we restrict ourselves to studying the spectrum of $\mathcal{K}$.

3. Preliminary description of the results. Since the domain $\Omega$ is unbounded, the embedding $H^{1}(\Omega) \subset L_{2}(\Omega)$ is not compact, so that the essential spectrum of $\mathcal{K}$ consists of more than the point $\mu=0$ (see [11, Theorem 9.2.1]). In $\S 2$ we use the Gelfand and Fourier transformations to introduce model problems on the periodicity cell $\Pi$ and on the section $\Theta=(-1 / 2,1 / 2) \times(-l / 2, l / 2)$ of its cylindrical part, which determine the structure of the essential spectrum. Largely, we use the method traditional for the theory of elliptic boundary-value problems in domains with piecewise smooth boundary 
(see the key publications [12]-15] and, e.g., the books [16, 17]), which include, as an intermediate element, the study of systems of differential equations in domains that go to infinity cylindrically or quasicylindrically (periodically). That theory provides necessary and sufficient conditions for the operator of the boundary-value problem in question to be Fredholm in the usual or weighted Sobolev spaces. The loss of the Fredholm property for some $\lambda$ means precisely the arising of a point (1.15) that belongs to the essential spectrum of $\mathcal{K}$.

Nevertheless, when studying the operator of problem (1.4)-(1.6), we need to deviate from the usual method. First, since the data of the problem are not sufficiently smooth, we are forced to deal with the variational setting (1.12) (rather than with the classical one, as is practical in the theory in question). However, this does not cause any complications, because we can use the Parseval identities for both transformations. Though such a generalization is evident, the author has found no detailed account of the corresponding arguments in the literature. They are reproduced in the proofs of Theorems 2.1 and 2.2 , but not in detail.

A more substantial deviation from the standard pattern is caused by the unboundedness of the periodicity cell $\Pi$ : the pencil $\mathfrak{A}$ related to the model problem (2.6) on $\Pi$ (see formula (2.7)) acquires both the discrete spectrum (2.36) and the continuous spectrum. In its turn, the continuous spectrum of the operator $\mathcal{K}$ is born by both parts of the spectrum of $\mathfrak{A}$. This gives rise to the absolute threshold $\mu_{\dagger}^{0}$ and to the partial thresholds $\mu_{\dagger}^{(n)}$ (see formulas (2.50) and (2.51)), while the essential spectrum is obtained as the union of the segments $\left[0, \mu_{\dagger}^{0}\right]$ and $\Delta^{(n)}$ corresponding to the above thresholds (see (2.52)). This structure gives way to arising gaps, i.e., intervals containing points of the discrete spectrum only, but having both its ends in the essential spectrum. The verification that such a gap arises indeed in the case of a "cushion with fringe" depicted schematically in Figures 5 and 6 , is the main result of the present paper. To deduce estimates for the eigenvalues of the model problem on the cell $\Pi$ (Figures 3,4 ) we apply the max-min principle (see, e.g., 11, Theorem 10.2.2]), along with asymptotic constructions typical of joints of elastic bodies that have different limiting dimensions (see, e.g., [18]-[21]).

The model problem on the periodicity cell involves two parameters: the spectral parameter $\lambda$ occurring in the system of differential equations (1.4), and the dual variable $\eta$ of the Gelfand transformation. In the above-mentioned theory of boundary-value problems in domains with piecewise smooth boundary, the parameter $\lambda$ is fixed, and, when the spectrum of the quadratic pencil (2.7) generated by the model problem in $\Pi$ is studied, some complex values of the prescribed spectral parameters $\eta$ are allowed. Another method for the investigation of formally selfadjoint systems of differential equations in $\mathbb{R}^{n}$ with periodic coefficients is based on the theory of selfadjoint operators in Hilbert space (see the survey [22] and the books [24, 25]); in the framework of that method, the dual variable $\eta \in \mathbb{R}^{n}$ is fixed, and the object of study becomes the spectrum of the unbounded operator generated by the corresponding Hermitian form (see [11, Chapter 10]) in the Lebesgue space on the periodicity cell. In this paper we use both approaches; in particular, the band structure of the spectrum is established via describing the spectrum of the problem operator on the cell.

It should be noted that the known examples of a gap opening in the continuous spectrum pertain precisely to the setting of elliptic systems with periodic coefficients in $\mathbb{R}^{n}$, as described in the preceding paragraph (see the papers [23], 26]-30] and the survey [31]), and the result is achieved with the help of the method of variation of periodic coefficients. Below, a gap in the continuous spectrum of the operator of problem (1.4)(1.6) will be constructed exclusively by choosing the form of the cell; i.e., the matrix $A^{0}$ of elastic modules and the density $\rho$ may be thought of as constants. 


\section{§2. THE BASIC PROPERTIES OF THE OPERATOR}

1. The model problem and the Gelfand transformation. We freeze the coefficients of our differential operators at infinity, i.e., we make the changes $A(x) \mapsto A^{0}$ and $\rho(x) \mapsto$ $\rho_{0}$ (see the stabilization conditions (1.10) ), and pass from the domain $\Omega$ to the periodic set $\Omega_{0}$ (see formula (1.3)). Then, the Neumann conditions (intrinsic conditions in the terminology of (1.5)) in the resulting model problem,

$$
\left(A^{0} D\left(\nabla_{x}\right) u, D\left(\nabla_{x}\right) v\right)_{\Omega_{0}}=\lambda \rho_{0}(u, v)_{\Omega_{0}}, \quad v \in \mathcal{H}_{0},
$$

are imposed on the surface $\Sigma_{0}=\partial \Omega_{0} \backslash \bar{\Gamma}_{0}$, and the (stable) Dirichlet conditions (1.6) are imposed on the surface $\Gamma_{0}^{ \pm}$described in (1.11) and having periodic structure. Accordingly, the Hilbert space $\mathcal{H}_{0}$ consists of vector-valued functions $u \in H^{1}\left(\Omega_{0}\right)^{3}$ that vanish for $x \in \Gamma_{0}=\Gamma_{0}^{+} \cup \Gamma_{0}^{-}$. We recall that $(u, v)_{\Omega_{0}}$ is the inner product in $L_{2}\left(\Omega^{0}\right)$.

The Gelfand transformation (discrete Fourier transformation; see 32] and also, e.g., the books [25, 16] and the paper [33]), defined by the formula

$$
v(x) \mapsto \widehat{v}(x ; \eta)=\frac{1}{\sqrt{2 \pi}} \sum_{j \in \mathbb{Z}} \exp \left(-i \eta\left(j+y_{1}\right)\right) v\left(j+y_{1}, y_{2}, z\right),
$$

yields the isometric isomorphism

$$
L_{2}\left(\Omega_{0}\right) \cong L_{2}\left(0,2 \pi ; L_{2}(\Pi)\right)
$$

Here $L_{2}(0,2 \pi ; \mathfrak{B})$ is the space of abstract functions with values in a Banach space $\mathfrak{B}$, and the norm looks like this:

$$
\left\|v ; L_{2}(0,2 \pi ; \mathfrak{B})\right\|=\left(\int_{0}^{2 \pi}\|v(\eta) ; \mathfrak{B}\|^{2} d \eta\right)^{1 / 2} .
$$

Observe that on the left-hand side in (2.2) the point $x$ belongs to the periodic domain $\Omega_{0}$, while on the right-hand side $x$ is an element of the standard periodicity cell $\Pi$ (see the definition in (1.30).

The inverse transformation is of the form

$$
\widehat{v}(x ; \eta) \mapsto v(x)=\frac{1}{\sqrt{2 \pi}} \int_{0}^{2 \pi} \exp \left(i \eta y_{1}\right) \widehat{v}\left(y_{1}-\left[y_{1}\right], y_{2}, z ; \eta\right) d \eta
$$

Here $[t]=\max \{n \in \mathbb{Z}: n \leq t\}$ is the integral part of $t \in \mathbb{R}$ and, as usual, $i$ is the imaginary unit. Now $x \in \Pi$ on the left-hand side of (2.3), but $x \in \Omega_{0}$ on the right-hand side. Since $\widehat{v}\left(0, y_{2}, z\right)=\widehat{v}\left(1, y_{2}, z\right)$ for smooth functions with compact support, and

$$
\widehat{P v}(x ; \eta)=P\left(\partial_{1}+i \eta, \partial_{2}, \partial_{3}\right) \widehat{v}(x ; \eta)
$$

for any differential operator $P\left(\nabla_{x}\right)$ with constant (or periodic) coefficients, the transformation (2.2) establishes the isomorphism

$$
H^{1}\left(\Omega_{0}\right) \approx L_{2}\left(0,2 \pi ; H_{\mathrm{per}}^{1}(\Pi)\right) ;
$$

here $H_{\text {per }}^{1}(\Pi)$ is the Sobolev space of functions with period 1 in the variable $x_{1}=y_{1}$.

Using (2.4) and the Parseval identity

$$
(u, v)_{\Omega_{0}}=\int_{0}^{2 \pi}(\widehat{u}(\cdot ; \eta), \widehat{v}(\cdot, \eta))_{\Pi} d \eta,
$$

we reshape problem (2.1) to the following family of problems on the periodicity cell $\Pi$ :

$$
\begin{aligned}
\mathbf{q}(\mathbf{u}, \mathbf{v} ; \eta, \Pi) & :=\left(A^{0} D\left(\partial_{1}+i \eta, \partial_{2}, \partial_{3}\right) \mathbf{u}(\cdot ; \eta), D\left(\partial_{1}+i \bar{\eta}, \partial_{2}, \partial_{3}\right) \mathbf{v}(\cdot ; \eta)\right)_{\Pi} \\
& =\lambda \rho_{0}(\mathbf{u}(\cdot ; \eta), \mathbf{v}(\cdot ; \eta))_{\Pi}, \quad \mathbf{v} \in \stackrel{H}{\mathrm{per}}^{1}(\Pi ; \gamma)^{3}, \quad \text { for a.e. } \eta \in[0,2 \pi) .
\end{aligned}
$$


Note that, since in what follows we shall need complex values of the parameter $\eta$, on the left-hand side in (2.6) we write the complex conjugate number $\bar{\eta}$. Also in (2.6) $\stackrel{\circ}{H}_{\text {per }}^{1}(\Pi ; \gamma)$ denotes the Sobolev space of functions that vanish on $\gamma=\Gamma_{0} \cap \partial \Pi$ and have period 1 in the variable $y_{1}$, and for simplicity of notation the Gelfand transforms are denoted by letters in bold.

Remark 2.1. The Parseval identity (2.5) and the extension of the Lebesgue inner product by duality between the Sobolev space $H^{1}\left(\Omega_{0}\right)$ and its dual $H\left(\Omega_{0}\right)^{*}$ allow us to extend the Gelfand transformation to functionals in $H^{1}\left(\Omega_{0}\right)^{*}$ and to establish the isomorphism

$$
H^{1}\left(\Omega_{0}\right)^{*} \approx L_{2}\left(0,2 \pi ; H_{\mathrm{per}}^{1}(\Pi)^{*}\right) .
$$

This fact will be used in the study of the nonhomogeneous problems (2.1) and (2.6).

By the Riesz theorem on the representation of a linear functional in Hilbert space, the variational spectral problem (2.6) gives rise to a quadratic pencil

$$
\eta \mapsto \mathfrak{a}(\eta ; \lambda): \stackrel{\circ}{H}_{\text {per }}^{1}(\Pi ; \gamma)^{3} \rightarrow \stackrel{\circ}{\text { per }}_{1}^{1}(\Pi ; \gamma)^{3} .
$$

Since the domain $\Pi$ is unbounded, this pencil requires a separate consideration.

2. Spectrum of the pencil on a cross section of the periodicity cell. For fixed $\eta \in[0,2 \pi)$ and $\lambda \in \mathbb{R}$, we apply the Fourier transformation

$$
\mathbf{v}(x ; \eta) \mapsto V(y ; \eta, \zeta)=\frac{1}{\sqrt{2 \pi}} \int_{\mathbb{R}} \exp (-i z \zeta) \mathbf{v}(y, z ; \eta) d z
$$

to problem (2.6) after multiplying its solution by an appropriate cutoff function. This yields yet another family of spectral problems on the rectangle $\Theta=(-1 / 2,1 / 2) \times$ $(-l / 2, l / 2)$, which is a cross section of the prism $\Pi$. Namely,

$$
\begin{array}{r}
Q(U, V ; \eta, \zeta):=\left(A^{0} D\left(\partial_{1}+i \eta, \partial_{2}, i \zeta\right) U(\cdot ; \eta, \zeta), D\left(\partial_{1}+i \bar{\eta}, \partial_{2}, i \bar{\zeta}\right) V(\cdot ; \eta, \zeta)\right)_{\Theta} \\
=\lambda \rho^{0}(U(\cdot ; \eta, \zeta), V(\cdot ; \eta, \zeta))_{\Theta}, \quad V \in \stackrel{H}{\mathrm{per}}^{1}(\Theta ; \theta)^{3}, \quad \text { for } \quad \text { a.e. } \zeta \in \mathbb{R}
\end{array}
$$

where $\stackrel{\circ}{H}_{\text {per }}^{1}(\Theta ; \theta)$ is the Sobolev space of functions 1-periodic with respect to $y_{1}$ and vanishing on the sides $\theta^{ \pm}=(-1 / 2,1 / 2) \times\{ \pm l / 2\}$ of the rectangle, and the complex conjugate parameters $\eta$ and $\zeta$ arise for the same reason as in (2.6). Problem (2.9) has its own quadratic pencil

$$
\zeta \mapsto \mathfrak{A}(\zeta ; \eta, \lambda): \stackrel{\circ}{H}_{\text {per }}^{1}(\Theta ; \theta)^{3} \rightarrow \stackrel{\circ}{H}_{\text {per }}^{1}(\Theta ; \theta)^{3} .
$$

We recall that the parameters $\eta$ and $\lambda$ are assumed to be fixed and, so far, real.

The following facts should be mentioned. First, the coefficients of the differential operators involved are constant, and the periodicity conditions make the angular points on the boundary of the rectangle inessential. Thus, the variational setting (2.9) is equivalent to the classical one (see the results on smoothness improvement for solutions of elliptic boundary-value problems in [10, Chapter 2]):

$$
\begin{aligned}
L\left(\nabla_{y}, i \zeta ; i \eta\right) U(y ; \eta, \zeta) & :=D\left(-\partial_{1}-i \eta,-\partial_{2},-i \zeta\right)^{\top} A^{0} D\left(\partial_{1}+i \eta, \partial_{2}, i \zeta\right) U(y ; \eta, \zeta) \\
& =\lambda \rho^{0} U(y ; \eta, \zeta), \quad y \in \Theta, \quad U(y ; \eta, \zeta)=0, \quad y \in \theta^{ \pm}, \\
U\left(\frac{1}{2}, y_{2} ; \eta, \zeta\right) & =U\left(-\frac{1}{2}, y_{2} ; \eta, \zeta\right), \\
\partial_{1} U\left(\frac{1}{2}, y_{2} ; \eta, \zeta\right) & =\partial_{1} U\left(-\frac{1}{2}, y_{2} ; \eta, \zeta\right), \quad\left|y_{2}\right|<\frac{l}{2} .
\end{aligned}
$$

As a result, we can view the pencil (2.10) as a map

$$
\mathfrak{A}(\zeta ; \eta, \lambda): H_{\text {per }}^{s+1}(\Theta)^{3} \cap \stackrel{\circ}{H}_{\text {per }}^{1}(\Theta ; \theta)^{3} \rightarrow H_{\text {per }}^{s-1}(\Theta)^{3}
$$

for any smoothness exponent $s \in \mathbb{N}=\{1,2, \ldots\}$. 
Second, the embedding $H^{1}(\Theta) \rightarrow L_{2}(\Theta)$ is compact, and for any $\zeta_{1}, \zeta_{2} \in \mathbb{C}$ the difference $L\left(\nabla_{y} ; \eta, \zeta_{1}\right)-L\left(\nabla_{y} ; \eta, \zeta_{2}\right)$ turns out to be a matrix first order differential operator (see the definition in the first line of (2.11)); i.e., $\mathfrak{A}\left(\zeta_{1} ; \eta, \Lambda\right)-\mathfrak{A}\left(\zeta_{2} ; \eta, \Lambda\right)$ is a compact operator. Consequently, by [34, 1.5.1], the map (2.12) is an isomorphism for all $\zeta \in \mathbb{C}$ except for a countable collection of normal eigenvalues with a single accumulation point at infinity (see also Remark 2.2).

Finally, $L\left(\nabla_{y}, i \zeta ; \eta\right)$ is an elliptic differential operator with a parameter (see [35]), i.e.,

$$
\operatorname{det} L(\xi, i \zeta ; \eta) \geq c_{\eta}\left(|\xi|^{2}+|\zeta|^{2}\right), \quad \xi \in \mathbb{R}^{2}, \quad \zeta \in \mathbb{R} .
$$

Therefore, by the results of [35], the eigenvalues of the pencil $\mathfrak{A}$ lie inside the union of a strip and a double angle:

$$
\left\{\zeta \in \mathbb{C}:|\operatorname{Im} \zeta| \leq \beta_{\eta, \lambda}\right\} \cup\left\{\zeta \in \mathbb{C}:|\operatorname{Re} \zeta| \leq \varkappa_{\eta, \lambda}|\operatorname{Im} \zeta|\right\} .
$$

The positive numbers $c_{\eta}$ and $\beta_{\eta, \lambda}, \varkappa_{\eta, \lambda}$ in (2.13) and (2.14) depend on $\eta$ and $\lambda$, but obvious reasons show that they can be taken to be the same for all $\eta \in[0,2 \pi)$.

Remark 2.2. If $\eta, \lambda \in \mathbb{R}$ and $i \zeta \in \mathbb{R},|\zeta|>\beta_{\eta, \lambda}$, then the formally selfadjoint problem (2.11) is uniquely solvable, because $\zeta$ does not belong to the set (2.11) and, thus, is not an eigenvalue of [34]. This observation establishes one of the conditions of Theorem 1.5.1 in 34] (the other conditions have already been verified).

3. Spectrum of the pencil on the periodicity cell. The following statement about the solvability of the problem

$$
q(\mathbf{u}, \mathbf{v} ; \eta, \Pi)-\lambda \rho_{0}(\mathbf{u}, \mathbf{v})_{\Pi}=\mathbf{f}(\mathbf{v}), \quad \mathbf{v} \in \stackrel{\circ}{H}_{\mathrm{per}}^{1}(\Pi ; \gamma)^{3},
$$

with a linear functional $\mathbf{f} \in\left(\stackrel{\circ}{H}_{\text {per }}^{1}(\Pi ; \gamma)^{3}\right)^{*}$ is verified by a standard method (see [12] and also [16]).

Theorem 2.1. Let $\eta \in[0,2 \pi)$. The operator of problem (2.15), viewed as the map (2.7), is Fredholm if and only if the pencil (2.12) has no real eigenvalues.

Proof. The "if" part. By the Korn inequality (3.1) (to be discussed below), the auxiliary problem

$$
q\left(\mathbf{u}^{H}, \mathbf{v}^{H} ; \eta, \Pi^{H}\right)-\left(\lambda-\lambda_{H}\right) \rho_{0}\left(\mathbf{u}^{H}, \mathbf{v}^{H}\right)_{\Pi^{H}}=\mathbf{f}^{H}\left(\mathbf{v}^{H}\right), \mathbf{v}^{H} \in \stackrel{\circ}{H}_{\text {per }}^{1}\left(\Pi^{H} ; \gamma^{H}\right)^{3},
$$

posed on the prism $\Pi^{H}=\{x \in \Pi: z>-4 H\}$ (see the definition (1.2)) and involving a large positive number $\lambda_{H}$, is uniquely solvable for any functional $\mathbf{f}^{H}$ on the Sobolev space $\stackrel{\circ}{\text { per }}^{1}\left(\Pi^{H} ; \gamma^{H}\right)^{3}$ of vector-valued functions in $H^{1}\left(\Pi^{H}\right)^{3}$ that vanish additionally on the surface $\gamma^{H}=\left(\partial \Pi^{H} \cap \Gamma_{0}\right) \cup\{x \in \Pi: z=-4 H\}$ and have period 1 in the variable $y_{1}$.

We construct a right regularizer (parametrix) for the operator of problem (2.15). The solution $\mathfrak{u} \in \stackrel{\circ}{H}_{\text {per }}^{1}(\Pi ; \gamma)^{3}$ of $(2.15)$ with a right-hand side $\mathfrak{f} \in\left(\stackrel{\circ}{H}_{\text {per }}^{1}(\Pi ; \gamma)^{3}\right)^{*}$ will be sought in the form

$$
\mathfrak{u}=\chi_{H}^{+1} \mathbf{u}^{H}+\left(1-\chi_{H}^{-1}\right) \mathbf{u}^{\infty},
$$

where $\chi_{H}^{p} \in C^{\infty}(\mathbb{R})$ is a cutoff function equal to 0 for $z \leq-(3+p) H$ and to 1 for $z \geq-(2+p) H$ (we use the indices $p= \pm 1$ and $p=0$ ). As components in (2.17), we take the solution $\mathbf{u}^{H} \in \stackrel{\circ}{H}_{\mathrm{per}}^{1}\left(\Pi^{H} ; \gamma^{H}\right)^{3}$ of problem (2.16), extended by zero to $\Pi^{H}$, and the solution $\mathbf{u}^{\infty} \in \stackrel{\circ}{H}_{\text {per }}^{1}\left(\Pi^{\infty} ; \gamma^{\infty}\right)^{3}$ of the following problem in the infinite prism $\Pi^{\infty}=(-1 / 2,1 / 2) \times(-l / 2, l / 2) \times \mathbb{R}:$

$$
q\left(\mathbf{u}^{\infty}, \mathbf{v}^{\infty} ; \eta, \Pi^{\infty}\right)-\lambda \rho_{0}\left(\mathbf{u}^{\infty}, \mathbf{v}^{\infty}\right)_{\Pi^{\infty}}=\mathbf{f}^{\infty}\left(\mathbf{v}^{\infty}\right), \quad \mathbf{v}^{\infty} \in \stackrel{\circ}{H}_{\text {per }}^{1}\left(\Pi^{\infty}, \gamma^{\infty}\right)^{3} .
$$

The right-hand sides of the above problems will be the functionals $\mathbf{f}^{H}(v)=\mathfrak{f}\left(\chi_{H}^{0} v\right)$ and $\mathbf{f}^{\infty}(v)=\mathfrak{f}\left(\left(1-\chi_{H}^{0}\right) v\right)$, respectively, and the Dirichlet conditions for (2.18) will be imposed 
on the union $\gamma^{\infty}$ of the strips $\gamma_{ \pm}^{\infty}=(-1 / 2,1 / 2) \times\{ \pm l / 2\} \times \mathbb{R}$. The presence of the cutoff function $\chi_{H}^{0}$ ensures that $\mathbf{f}^{H} \in\left(\stackrel{\circ}{H}_{\text {per }}^{1}\left(\Pi^{H} ; \gamma^{H}\right)^{3}\right)^{*}$. Hence, there is a vector-valued function $\mathbf{u}^{H} \in \stackrel{\circ}{H}_{\text {per }}^{1}\left(\Pi^{H} ; \gamma^{H}\right)^{3}$ satisfying the integral identity (2.16) and such that

$$
\left\|\mathbf{u}^{H} ; \stackrel{\circ}{H}_{\text {per }}^{1}\left(\Pi^{H} ; \gamma^{H}\right)\right\| \leq c_{H}\left\|\mathbf{f}^{H} ; \stackrel{\circ}{H}_{\text {per }}^{1}\left(\Pi^{H} ; \gamma^{H}\right)^{*}\right\| \leq C_{H}\left\|\mathfrak{f} ; \stackrel{\circ}{H}_{\text {per }}^{1}(\Pi ; \gamma)^{*}\right\| .
$$

To find a solution of (2.18), we apply the Fourier transformation (2.8). The Parseval identity for Fourier images reshapes (2.9) to a family of nonhomogeneous problems (2.9):

$$
\begin{aligned}
& \left(A^{0} D\left(\partial_{1}+i \eta, \partial_{2}, i \zeta\right) U^{\infty}(y ; \eta, \zeta), D\left(\partial_{1}+i \eta, \partial_{2}, i \zeta\right) V^{\infty}(\cdot ; \eta, \zeta)\right)_{\Theta} \\
& \quad-\lambda \rho^{0}\left(U^{\infty}(\cdot ; \eta, \zeta), V^{\infty}(\cdot ; \eta, \zeta)\right)_{\Theta}=F\left(V^{\infty} ; \eta, \zeta\right), \quad V^{\infty} \in \stackrel{\circ}{\mathrm{per}}_{1}^{1}(\Theta ; \theta)^{3} .
\end{aligned}
$$

Since the line $\mathbb{R} \subset \mathbb{C}$ is free from the spectrum of the pencil $\mathfrak{A}$, the estimates [35] for solutions of elliptic problems with a parameter (see [12] or [16, Chapter 3] for the details) show that a solution $U^{\infty} \in \stackrel{\circ}{H}_{\text {per }}^{1}(\Theta ; \theta)^{3}$ of problems (2.20) exists for a.e. $\zeta \in \mathbb{R}$, and

$$
\left\|U^{\infty} ; \Theta\right\|_{\zeta} \leq c\left\|F^{\infty} ; \Theta\right\|_{\zeta *},
$$

where $c$ is independent of the functional $F^{\infty}$ and the parameter $\zeta$, and

$$
\begin{aligned}
\left\|U^{\infty} ; \Theta\right\| \|_{\zeta}^{2} & =\left\|\nabla_{y} U^{\infty} ; L_{2}(\Theta)\right\|^{2}+\left(1+\zeta^{2}\right)\left\|U^{\infty} ; L_{2} \Theta\right\|^{2}, \\
\left\|F^{\infty} ; \Theta\right\|_{\zeta *} & =\sup \left\{\left|F^{\infty}\left(V^{\infty}\right)\right|: V^{\infty} \in \stackrel{\circ}{\mathrm{per}}^{1}(\Theta ; \theta)^{3},\left\|V^{\infty} ; \Theta\right\|_{\zeta}=1\right\} .
\end{aligned}
$$

Now, the inverse Fourier transformation

$$
U^{\infty}(y ; \eta, \zeta) \mapsto \mathbf{u}^{\infty}(x ; \eta)=\frac{1}{\sqrt{2 \pi}} \int_{\mathbb{R}} \exp (i z \zeta) U^{\infty}(y ; \eta, \zeta) d \zeta
$$

provides a solution $\mathbf{u}^{\infty} \in \stackrel{\circ}{H}_{\mathrm{per}}^{1}\left(\Pi^{\infty} ; \gamma^{\infty}\right)^{3}$ of problem (2.18), while formulas (2.21) and (2.22), together with the rule for differentiation of Fourier images, ensure the estimate

$$
\begin{aligned}
\left\|\mathbf{u}^{\infty} \stackrel{\circ}{\mathrm{per}}_{\mathrm{per}}^{1}\left(\Pi^{\infty} ; \gamma^{\infty}\right)\right\|^{2} & \leq \int_{\mathbb{R}}\left\|U^{\infty} ; \Theta\right\|_{\zeta}^{2} d \zeta \leq c \int_{\mathbb{R}}\left\|F^{\infty} ; \Theta,\right\| \|_{\zeta *}^{2} d \zeta \\
& \leq c\left\|\mathbf{f}^{\infty} ; \stackrel{\circ}{\mathrm{per}}^{1}\left(\Pi^{\infty} ; \gamma^{\infty}\right)\right\|^{2} \leq c\left\|\mathfrak{f} ; \stackrel{\circ}{\mathrm{per}}^{1}(\Pi ; \gamma)\right\|^{2} .
\end{aligned}
$$

The last inequalities in the chains (2.23) and (2.19) are ensured by the properties of the functionals $\mathbf{f}^{\infty}$ and $\mathbf{f}^{H}$.

We calculate the discrepancy of the vector-valued function (2.17) in problem 2.15) with a right-hand side $\mathbf{f}$. Let $\mathbf{v} \in \stackrel{\circ}{H}_{\text {per }}^{1}(\Pi ; \gamma)^{3}$, and take $\mathbf{v}^{H}=\chi_{H}^{+1} \mathbf{v}$ and $\mathbf{v}^{\infty}=\left(1-\chi_{H}^{-1}\right) \mathbf{v}$ as test functions in (2.16) and (2.18), respectively. Observe that

$$
\mathfrak{f}(\mathbf{v})=\mathbf{f}^{H}\left(\mathbf{v}^{H}\right)+\mathbf{f}^{\infty}\left(\mathbf{v}^{\infty}\right)
$$

by the definitions of the cutoff functions and the right-hand sides of the integral identities. Also, we have

$$
\begin{aligned}
q\left(\mathbf{u}^{H}, \mathbf{v}^{H} ; \eta, \Pi^{H}\right) & =q\left(\chi_{H}^{+1} \mathbf{u}^{H}, \mathbf{v} ; \eta, \Pi\right)+\widetilde{q}^{H}\left(\mathbf{u}^{H}, \mathbf{v} ; \eta, \Pi\right), \\
q\left(\mathbf{u}^{\infty} \mathbf{v}^{\infty} ; \eta, \Pi^{\infty}\right) & =q\left(\left(1-\chi_{H}^{-1}\right) \mathbf{u}^{\infty}, \mathbf{v} ; \eta, \Pi\right)+\widetilde{q}^{\infty}\left(\mathbf{u}^{\infty}, \mathbf{v} ; \eta, \Pi\right),
\end{aligned}
$$

where

$$
\begin{aligned}
\mathcal{F}^{H}(\mathfrak{f}, \mathbf{v}):=\widetilde{q}^{H}\left(\mathbf{u}^{H}, \mathbf{v} ; \eta, \Pi\right)= & \left(A^{0} D\left(\partial_{1}+i \eta, \partial_{2}, \partial_{3}\right) \mathbf{u}^{H}, D\left(0,0, \partial_{3} \chi_{H}^{+1}\right) \mathbf{v}\right)_{\Pi} \\
& -\left(A^{0} D\left(0,0, \partial_{3} \chi_{H}^{+1}\right) \mathbf{u}^{H}, D\left(\partial_{1}+i \eta, \partial_{2}, \partial_{3}\right) \mathbf{v}\right)_{\Pi} \\
\mathcal{F}^{\infty}(\mathfrak{f}, \mathbf{v}):=\widetilde{q}^{\infty}\left(\mathbf{u}^{\infty}, \mathbf{v} ; \eta, \Pi\right)= & -\left(A^{0} D\left(\partial_{1}+i \eta, \partial_{2}, \partial_{3}\right) \mathbf{u}^{\infty}, D\left(0,0, \partial_{3} \chi_{H}^{-1}\right) \mathbf{v}\right)_{\Pi} \\
& +\left(A^{0} D\left(0,0, \partial_{3} \chi_{H}^{-1}\right) \mathbf{u}^{\infty}, D\left(\partial_{1}+i \eta, \partial_{2}, \partial_{3}\right) \mathbf{v}\right)_{\Pi}
\end{aligned}
$$


The forms (2.24) arose as a result of shifting the cutoff functions $\chi_{H}^{+1}$ and $1-\chi_{H}^{-1}$ from the test function $\mathbf{v}$ to the solutions $\mathbf{u}^{H}$ and $\mathbf{u}^{\infty}$. While doing so, we were forced to commutate twice the differential operator $D\left(\partial_{1}+i \eta, \partial_{2}, \partial_{3}\right)$ with the cutoff functions mentioned above, obtaining the matrix-valued functions $D\left(0,0, \partial_{3} \chi_{H}^{+1}\right)$ and $D\left(0,0,-\partial_{3} \chi_{H}^{-1}\right)$ (observe that $\chi_{H}^{p}$ depends only on the variable $\left.x_{3}=z\right)$. In what follows the fact that the supports of the derivatives $\partial_{3} \chi_{H}^{+1}$ and $\partial_{3}\left(1-\chi_{H}^{-1}\right)=-\partial_{3} \chi_{H}^{-1}$ are compact sets will be important.

So, adding the integral identities (2.16) and (2.18), making the above transformations, and recalling the definitions (2.17), we find that

$$
q(\mathfrak{u}, \mathbf{v} ; \eta, \Pi)-\lambda \rho^{0}(\mathfrak{u}, \widehat{\mathbf{v}})_{\Pi}=\mathfrak{f}(\mathbf{v})-\mathcal{F}^{H}(\mathfrak{f}, \mathbf{v})-\mathcal{F}^{\infty}(\mathfrak{f}, \widehat{\mathbf{v}})-\mathcal{F}^{\lambda}(\mathfrak{f}, \mathbf{v}) .
$$

The linear maps

$$
\left(\stackrel{\circ}{H}_{\text {per }}^{1}(\Pi ; \gamma)^{3}\right)^{*} \ni \mathfrak{f} \mapsto \mathcal{F}^{H}(\mathfrak{f}, \cdot), \mathcal{F}^{\infty}(\mathfrak{f}, \cdot), \mathcal{F}^{\lambda}(\mathfrak{f}, \cdot) \in\left(\stackrel{\circ}{H}_{\text {per }}^{1}(\Pi ; \gamma)^{3}\right)^{*}
$$

turn out to be compact, because (2.24) and the expression

$$
\mathcal{F}^{\lambda}(\mathfrak{f}, \widehat{\mathbf{v}})=\lambda_{H} \rho_{0}\left(\mathbf{u}^{H}, \chi_{H}^{+1} \mathbf{v}\right)_{\Pi^{H}}
$$

involve no products of first derivatives of components of the vector-valued functions $\mathbf{u}^{H}, \mathbf{u}^{\infty}$ and $\mathbf{v}$, and integration in all cases is over the bounded set $\left\{x \in \Pi^{\infty}:-H>z>\right.$ $-4 H\}$. Thus, for the abstract equation

$$
\mathfrak{f}-\mathcal{F}^{H}(\mathfrak{f}, \cdot)-\mathcal{F}^{\infty}(\mathfrak{f},, \cdot)-\mathcal{F}^{\lambda}(\mathfrak{f}, \cdot)=\mathbf{f} \in\left(\stackrel{\circ}{H}^{1}(\Pi ; \gamma)^{3}\right)^{*},
$$

the Fredholm alternative is valid; i.e., if the right-hand side $\mathbf{f}$ satisfies a finite collection of orthogonality conditions, then (2.26) admits a solution $\mathfrak{f}$ for which formula (2.25) turns into the integral identity (2.15), which has a solution (2.17). In other words, we have constructed a right regularizer; now, in order to check that the operator of problem (2.4) is Fredholm, it remains to verify that the kernel of the operator is finite-dimensional; this will be done in Remark 2.4.

The "only if" part. If the real axis $\mathbb{R}$ contains an eigenvalue of the pencil (2.12), then the range of the operator of problem (2.15), viewed as a map (2.7), is not closed. The corresponding singular sequence of functions for this operator can be constructed in a standard way (see [12] and, e.g., [16, Theorem 3.1.1 and Remark 3.1.5]). Moreover, in the proof of the second part of Theorem 2.2 we shall find a Weyl singular sequence for the operator $\mathcal{K}$ in a similar situation and by the same method. Therefore, here we do not present these constructions.

For $\eta \in[0,2 \pi)$ and $\zeta \in \mathbb{R}$, we introduce the unbounded symmetric operator $M(\eta, \zeta)$ generated in $L_{2}(\Theta)^{3}$ by the Hermitian form $Q$ defined in (2.9) (see [11, $\left.\S 10.1\right]$ ). The relationship between the pencils (2.10) and (2.12) (see above) shows that the domain $\mathcal{D}(M(\eta, \zeta))$ of $M(\eta, \zeta)$ is the subspace $H_{\text {per }}^{2}(\Theta)^{3} \cap \stackrel{\circ}{H}^{1}(\Theta ; \theta)^{3}$, and this operator is closed. It is selfadjoint, because the matrix differential operator $L\left(\nabla_{y}, i \zeta ; i \eta\right)$ in problem (2.11) is formally selfadjoint. Since the embedding $H^{1}(\Theta) \subset L_{2}(\Theta)$ is compact, Theorems 10.2.5 and 10.2.2 in [1] imply that the operator $M(\eta, \zeta)$ has a discrete spectrum consisting of eigenvalues

$$
0<\Lambda_{1}(\eta, \zeta) \leq \Lambda_{2}(\eta, \zeta) \leq \cdots \leq \Lambda_{n}(\eta, \zeta) \leq \cdots \rightarrow+\infty
$$

(we list them with multiplicities; the fact that they are positive is ensured by the lemma below). Moreover,

$$
\Lambda_{1}(\eta, \zeta)=\inf \left\{\left\|U ; L_{2}(\Theta)\right\|^{-2} Q(U, U ; \eta, \zeta) \mid U \in \stackrel{\circ}{H}_{\mathrm{per}}^{1}(\Theta ; \theta)^{3} \backslash\{0\}\right\} .
$$


Lemma 2.1. We have

$$
Q(U, U ; \eta, \zeta) \geq c_{A}\left(1+|\zeta|^{2}\right)\left\|U ; L_{2}(\Theta)\right\|^{2},
$$

where $c_{A}>0$ is a constant independent of $\eta \in[0,2 \pi), \zeta \in \mathbb{R}$, and $U \in \stackrel{\circ}{H}_{\text {per }}^{1}(\Theta ; \theta)^{3}$.

Proof. Put

$$
\mathcal{U}(x)=\exp \left(i \eta y_{1}+i \zeta z\right) U(y) .
$$

Clearly, we have $\mathcal{U} \in \stackrel{\circ}{H}^{1}(\Theta \times(0,1) ; \theta \times(0,1))^{3}$, but the periodicity of vector-valued functions is lost. Nevertheless, by the Dirichlet conditions on the faces $\theta \times(0,1)$ of the parallelepiped $\Theta \times(0,1)$, we have the Korn inequality

$$
\begin{aligned}
\left\|\mathcal{U} ; L_{2}(\Theta \times(0, H))\right\|^{2} & +\left\|\nabla_{x} \mathcal{U} ; L_{2}(\Theta \times(0, H))\right\|^{2} \\
& \leq c_{H}\left\|D\left(\nabla_{x}\right) \mathcal{U} ; L_{2}(\Theta \times(0, H))\right\|^{2}
\end{aligned}
$$

(see [36, 37, 6], and also relation (3.1)). In the second term on the left, we keep only the derivative $\partial_{z} U$ and perform differentiation and integration with respect to $z \in(0, H)$. As a result, we arrive at the estimate

$$
\left(1+|\zeta|^{2}\right)\left\|U ; L_{2}(\Theta)\right\|^{2} \leq c_{h}\left\|D\left(\partial_{1}+i \eta, \partial_{2}, i \zeta\right) U ; L_{2}(\Theta)\right\|^{2},
$$

as required.

Formulas (2.29) and (2.28) imply the inequality $\Lambda_{1}(\eta, \zeta) \geq c_{A}\left(1+|\zeta|^{2}\right)$. Since the eigenvalues depend continuously on the parameter $\zeta$ (see, e.g., [38, Chapter 7]), the function $\zeta \mapsto \Lambda_{1}(\eta, \zeta)$ takes all values starting with

$$
\Lambda_{\min }(\eta)=\min \left\{\Lambda_{1}(\eta, \zeta): \zeta \in \mathbb{R}\right\}>0
$$

and up to $+\infty$. Thus, by Theorem 2.1, the operator of problem (2.15), viewed as the map (2.7), is not Fredholm for

$$
\lambda \geq \rho_{0}^{-1} \Lambda_{\min }(\eta) .
$$

4. Spectrum of the operator $\mathcal{K}$. For $\eta \in[0,2 \pi)$ fixed, we introduce the selfadjoint unbounded operator $m(\eta)$ in $L_{2}(\Pi)^{3}$ generated (see [11, Chapter 10]) by the Hermitian form $q(\mathbf{u}, \mathbf{v} ; \eta, \Pi)$ indicated on the left-hand side of (2.6) and closed on the space $\stackrel{\circ}{H}_{\text {per }}^{1}(\Pi ; \gamma)^{3}$. The next statement says that this operator is positive.

Lemma 2.2. We have

$$
q(\mathbf{u}, \mathbf{u} ; \eta, \Pi) \geq C_{A, \Pi}\left\|\mathbf{u} ; L_{2}(\Pi)\right\|^{2},
$$

where $C_{A, \Pi}>0$ is independent of $\eta \in[0,2 \pi)$ and $\mathbf{u} \in \stackrel{\circ}{H}_{\text {per }}^{1}(\Pi ; \gamma)^{3}$.

Proof. As in the proof of Lemma 2.1. we introduce a vector-valued function $\mathcal{U}$ by formula (2.30), but now we put $\zeta=0$ and replace $U(y)$ with $\mathbf{u}(x)$. We apply the Korn inequality (2.31) on the sets $\{x \in \Pi: z>-H\}$ and $\mathbb{Q}_{n}=\Theta \times(-(n-1) H,-n H), n \in \mathbb{Z}$; the corresponding constants will be denoted by $c_{0}$ and $c_{H}$. Observe that $c_{H}$ does not depend on $n$, because the parallelepipeds $\mathbb{Q}_{n}$ are congruent. Summing the inequalities, we obtain

$$
\left\|\mathcal{U} ; H^{1}(\Pi)\right\|^{2} \leq c_{\Pi}\left\|D\left(\nabla_{x}\right) \mathcal{U} ; L_{2}(\Pi)\right\|^{2},
$$

where $c_{\Pi}=\max \left\{c_{0}, c_{H}\right\}$. Now, keeping on the left only the $L_{2}$-norm of the vector-valued function $\mathbf{U}$ itself, and taking the factor $\exp \left(i \eta y_{1}\right)$ into account, we get

$$
\left\|\mathbf{u} ; L_{2}(\Pi)\right\|^{2} \leq c_{\Pi}\left\|D\left(\partial_{1}+i \eta, \partial_{2}, \partial_{3}\right) \mathbf{u} ; L_{2}(\Pi)\right\|^{2} .
$$

Note that $D\left(\nabla_{x}\right) \mathcal{U}=\exp (i \eta y) D\left(\partial_{1}+i \eta, \partial_{2}, \partial_{3}\right) \mathbf{u}$, and $\exp (i \eta y)$ disappears when we calculate norms, because $\eta$ is real. Relation (2.35) turns into (2.33) because the matrix $A^{0}$ is positive definite. 
By Theorem 2.1 and Definition (2.32), the essential spectrum of the operator $m(\eta)$ is located on the ray $\left[\Lambda_{\min }(\eta),+\infty\right)$. The interval $\left(0, \Lambda_{\min }(\eta)\right)$ contains only points of its discrete spectrum:

$$
0<c_{A, \Pi} \leq \Lambda^{(1)}(\eta) \leq \Lambda^{(2)}(\eta) \leq \cdots \leq \Lambda^{(N(\eta))}(\eta) .
$$

The number $N(\eta)$ of eigenvalues, listed in (2.36) with multiplicities, depends on $\eta$; the cases of $N(\eta)=0$ and $N(\eta)=+\infty$ are not excluded.

Remark 2.3. In general, the function $\eta \mapsto N(\eta)$ is not continuous, because the eigenvalues $\Lambda^{(n)}(\eta)$ can run away from the interval $\left(0, \Lambda_{\min }(\eta)\right)$, disappearing inside the continuous spectrum $\left[\Lambda_{\min }(\eta), \infty\right)$ of the operator $m(\eta)$. Thus, at the discontinuity points of the function in question, at least one of the one-sided limits of the eigenvalues $\Lambda^{(N(\eta))}(\eta)$ is equal to $\Lambda_{\min }(\eta)$.

Theorem 2.2. A point $\mu \in(0,1]$ belongs to the discrete spectrum or to the resolvent set of the operator $\mathcal{K}$ if and only if for $\lambda=\mu^{-1}-1$ the half-interval $[0,2 \pi)$ is free of the spectrum of the pencil (2.7).

Proof. The "only if" part. As in the proof of the first statement in Theorem 2.1, we consider an auxiliary problem on the bounded domain $\Omega(R)=\Omega_{0} \cap \mathbb{B}_{R}$ :

$$
\left(A D\left(\nabla_{x}\right) u^{R}, D\left(\nabla_{x}\right) v^{R}\right)_{\Omega(R)}-\left(\lambda-\lambda^{R}\right)\left(\rho u^{R}, v^{R}\right)_{\Omega(R)}=f^{R}\left(v^{R}\right), \quad v^{R} \in \mathcal{H}(R) .
$$

The space $\mathcal{H}(R)$ consists of all vector-valued functions $v^{R} \in \stackrel{\circ}{H}_{\text {per }}^{1}\left(\Omega(R) ; \Gamma \cap \mathbb{B}_{R}\right)^{3}$ that vanish on $\partial \mathbb{B}_{R} \cap \Omega_{0}$, and the parameter $\lambda^{R}$ is assumed to be sufficiently large to ensure that the following is true: by the Riesz theorem on representation of a linear functional, the Korn inequality for $u^{R} \in \mathcal{H}(R)$ guarantees the unique solvability of problem (2.37) and the estimate

$$
\left\|u^{R} ; H^{1}(\Omega(R))\right\| \leq c\left\|f^{R} ; \mathcal{H}(R)^{*}\right\|
$$

with a constant $c$ independent of the parameter $R \geq R^{0}$ and the functional $f^{R}$.

We construct a right regularizer for the operator of the problem

$$
\left(A D\left(\nabla_{x}\right) u, D\left(\nabla_{x}\right) v\right)_{\Omega}-\lambda(\rho u, v)_{\Omega}=f(v), \quad v \in \mathcal{H} .
$$

The solution of (2.38) with right-hand side $\mathfrak{g} \in \mathcal{H}^{*}$ will be sought in the form

$$
\mathfrak{v}=\chi_{R}^{2} u^{R}+\left(1-\chi_{R}^{0}\right) u^{0},
$$

where $\chi_{R}^{p} \in C^{\infty}\left(\mathbb{R}^{3}\right)$ is a cutoff function equal to zero for $|x| \geq(2+p) R$ and to 1 for $|x| \leq(1+p) R$, and $\left|\nabla_{x} \chi_{R}^{p}(x)\right| \leq c R^{-1}, p=0,1,2$. Also in $(2.3), u^{R} \in \mathcal{H}(R)$ is the solution of (2.37) in a bounded domain, and $u^{0} \in \mathcal{H}_{0}$ is the solution of the following problem on the periodic set $\Omega_{0}$ :

$$
\left(A^{0} D\left(\nabla_{x}\right) u^{0}, D\left(\nabla_{x}\right) v^{0}\right) \Omega_{0}-\lambda\left(\rho u^{0}, v^{0}\right) \Omega_{0}=f^{0}\left(v^{0}\right), \quad v^{0} \in \mathcal{H}_{0} .
$$

The right-hand sides have the form $f^{R}\left(v^{R}\right)=\mathfrak{g}\left(\chi_{R}^{1} v^{R}\right)$ and $f^{0}\left(v^{0}\right)=\mathfrak{g}\left(\left(1-\chi_{R}^{1}\right) v^{0}\right)$, respectively. Note that $\left(1-\chi_{R}^{0}\right) u^{0}$ satisfies the boundary-value condition (1.6), due to the definition of the cutoff function $\chi_{R}^{0}$ and the requirement (1.11).

Since the half-interval $[0,2 \pi)$ is free from eigenvalues of the pencil $\mathfrak{A}$, it follows that, combining the Gelfand transformation, the inversion of the operator of the problem

$$
q\left(\widehat{u}^{0}, \mathbf{v} ; \eta, \Pi\right)-\lambda \rho_{0}\left(\widehat{u}^{0}, \mathbf{v}\right)_{\Pi}=\widehat{f}^{0}(\mathbf{v}), \quad \mathbf{v} \in \stackrel{\circ}{H}_{\text {per }}^{1}(\Pi ; \gamma)^{3},
$$

with parameter $\eta \in[0,2 \pi)$, and the inverse transformation (2.3), we obtain a solution of (2.40) subject to the estimate

$$
\begin{aligned}
\left\|u^{0} ; \mathcal{H}_{0}\right\|^{2} \leq c \int_{0}^{2 \pi}\left\|\widehat{u}^{0} ; H^{1}(\Pi)\right\|^{2} d \eta & \leq c \int_{0}^{2 \pi}\left\|\widehat{f}^{0} ;\left(\stackrel{\circ}{\mathrm{per}}_{1}^{1}(\Pi ; \gamma)^{3}\right)^{*}\right\|^{2} d \eta \\
& \leq c\left\|f^{0} ; \mathcal{H}_{0}^{*}\right\|^{2} \leq c R\left\|\mathfrak{g} ; \mathcal{H}^{*}\right\|^{2} .
\end{aligned}
$$


Note that here (and throughout the proof) $c$ means a constant independent of $R$, and that the additional factor $R$ arose on the right in the chain (2.41) because $\left|\nabla_{x} \chi_{R}^{p}(x)\right| \leq c R^{-1}$.

Let $v \in \mathcal{H}$; then $v^{R}=\chi_{R}^{2} v \in \mathcal{H}(R)$ and $v^{0}=\left(1-\chi_{R}^{0}\right) v \in \mathcal{H}_{0}$. We plug these two test functions in the integral identities (2.37) and (2.40), respectively. Adding the results, we observe that, by the identities $\chi_{R}^{1} \chi_{R}^{2}=\chi_{R}^{1},\left(1-\chi_{R}^{1}\right)\left(1-\chi_{R}^{0}\right)=1-\chi_{R}^{1}$ and the definitions of $f^{R}$ and $f^{0}$, we have

$$
f^{R}\left(\chi_{R}^{2} v\right)+f^{0}\left(\left(1-\chi_{R}^{0}\right) v\right)=\mathfrak{g}\left(\chi_{R}^{1} v\right)+\mathfrak{g}\left(\left(1-\chi_{R}^{1}\right) v\right)=\mathfrak{g}(v) .
$$

Finally, we use (2.39) to arrive at the integral identity

$$
\left(A D\left(\nabla_{x}\right) \mathfrak{v}, D\left(\nabla_{x}\right) v\right)_{\Omega}-\lambda(\rho \mathfrak{v}, v)_{\Omega}=\mathfrak{g}(v)+\mathfrak{G}^{R}(\mathfrak{g} ; v)+\mathfrak{G}^{0}(\mathfrak{g} ; v), \quad v \in \mathcal{H}
$$

where

$$
\begin{aligned}
\mathfrak{G}^{R}(\mathfrak{g} ; v)= & \left(A D\left(\nabla_{x}\right) u^{R}, D\left(\nabla_{x} \chi_{R}^{2}\right) v\right)_{\Omega} \\
& -\left(A D\left(\nabla_{x} \chi_{R}^{2}\right) u^{R}, D\left(\nabla_{x}\right) v\right)_{\Omega}-\lambda^{R}\left(\rho u^{R}, \chi_{R}^{2} v\right)_{\Omega}, \\
\mathfrak{G}^{0}(\mathfrak{g} ; v)= & -\left(A D\left(\nabla_{x}\right) u^{0}, D\left(\nabla_{x} \chi_{R}^{0}\right) v\right)_{\Omega}+\left(A D\left(\nabla_{x} \chi_{R}^{0}\right) u^{0}, D\left(\nabla_{x}\right) v\right)_{\Omega} \\
& +\left(\left(A-A^{0}\right) D\left(\nabla_{x}\right) u^{0}, D\left(\nabla_{x}\right)\left(\left(1-\chi_{R}^{0}\right) v\right)\right)_{\Omega} \\
& -\lambda\left(\left(\rho-\rho^{0}\right) u^{0},\left(1-\chi_{R}^{0}\right) v\right)_{\Omega} .
\end{aligned}
$$

As in the calculations in (2.24), here we have performed a commutation of the differential operator $D\left(\nabla_{x}\right)$ with the cutoff functions $\chi_{R}^{2}$ and $1-\chi_{R}^{0}$, i.e., $D\left(\nabla_{x} \chi_{R}^{p}\right)^{\top}$ is a matrix-valued function obtained from (1.8) by the substitutions $\partial / \partial x_{j} \mapsto \partial_{j} \chi_{R}^{p}$. Also, to the right-hand side of (2.44) we have placed the discrepancies that arose because of freezing the coefficients at infinity (see the deduction of the model problem (2.1) in Subsection 1 of $\S 2$ ). The stabilization condition (1.10) and inequality (2.41) imply that the norm of the operator generated in the space $\mathcal{H}^{*}$ by the terms indicated does not exceed $c R^{N} \exp \left(-\delta_{0} R\right)$. The presence of the exponential function above shows that the factors with power-like growth, which arose, e.g., on the right-hand side in (2.41), leave the majorant infinitely small as $R \rightarrow+\infty$. The other terms in (2.43) and (2.44) give rise only to compact operators in $\mathcal{H}^{*}$, because the corresponding expressions contain no products of first derivatives of (components of) $u^{R}, u^{0}$, and $v$, and integration is taken over the bounded set $\{x \in \bar{\Omega}: R \leq|x| \leq 4 R\} \supset \operatorname{supp}\left|\nabla_{x} \chi_{R}^{p}\right|$. Thus, if $R \geq R^{0}$ is sufficiently large, then for the equation

$$
\mathfrak{g}+\mathfrak{G}^{R}(\mathfrak{g} ; \cdot)+\mathfrak{G}^{0}(\mathfrak{g} ; \cdot)=f \in \mathcal{H}^{*},
$$

similar to (2.26), the Fredholm alternative is valid. Imposing a finite number of orthogonality conditions on the right-hand side $f$, we can find the functional $\mathfrak{g} \in \mathcal{H}^{*}$. Then we can use formula (2.39) to obtain a function $\mathfrak{v} \in \mathcal{H}$ satisfying the integral identity (2.38) (by (2.42) and (2.45)). So, we have constructed a right regularizer for the operator of problem (2.38).

Now we show that the $\operatorname{kernel} \operatorname{ker}(\mathcal{K}-\mu)$ is finite-dimensional. Let $u \in \mathcal{H}$ be a solution of (1.12). Since problem (2.37) in the bounded domain $\Omega(R)$ is uniquely solvable, we get the relation

$$
\left\|\chi_{R}^{1} u ; H^{1}(\Omega)\right\| \leq c\left\|u ; L_{2}(\Omega(R))\right\| .
$$

Here the right-hand side arose because of commutation of the corresponding differential operator with the cutoff function $\chi_{R}^{1}(\operatorname{cf} .(2.43))$. We multiply the solution by $1-\chi_{R}^{1}$, freeze the coefficients, and turn to the uniquely solvable model problem (2.40); this gives the estimate

$$
\left\|\left(1-\chi_{R}^{1}\right) u ; H^{1}(\Omega)\right\| \leq c\left(\left\|u ; L_{2}(\Omega(R))\right\|+\exp \left(-\delta_{0} R\right)\left\|u ; H^{1}(\Omega)\right\|\right) .
$$


The two inequalities above imply that for sufficiently large $R$ we have

$$
\left\|u ; H^{1}(\Omega)\right\| \leq C\left\|u ; L_{2}(\Omega(R))\right\|,
$$

which shows that the subspace $\operatorname{ker}(\mathcal{K}-\mu)$ is finite-dimensional. It remains to mention that, by the definitions (1.13) and (1.14), problem (2.38) is equivalent to the nonhomogeneous abstract equation (1.16) with the right-hand side $(1+\lambda)^{-1} \mathcal{F} \in \mathcal{H}$ and with the parameter (1.15).

Thus, the operator $\mathcal{K}-\mu$ is Fredholm; i.e., the point $\mu$ does not belong to the essential spectrum of $\mathcal{F}$.

The "if" part. First, suppose that the half-interval $[0,2 \pi)$ contains an eigenvalue $\eta_{0}$ of the pencil $\eta \mapsto \mathfrak{a}(\eta ; \lambda)$. Then there is a nontrivial vector-valued function $\mathbf{u} \in \stackrel{\circ}{H}_{\text {per }}^{1}(\Pi ; \gamma)^{3}$ satisfying problem (2.6) with the parameters $\eta_{0}$ and $\lambda$. Put

$$
u^{q}(x)=2^{-q / 2} X\left(y_{1}-2^{q}\right) X\left(2^{q+1}-y_{1}\right) \exp \left(i \eta_{0} y_{1}\right) \mathbf{u}(x) .
$$

Here $X \in C^{\infty}(\mathbb{R})$ is a cutoff function such that $X\left(y_{1}\right)=0$ for $y_{1} \leq 0, X\left(y_{1}\right)=1$ for $y_{1} \geq 1$, and $0 \leq X \leq 1$. The number $q \in \mathbb{N}$ is taken so large that the support $\left\{x: 2^{q} \leq y_{1} \leq 2^{q+1}\right\}$ of the vector-valued function $u^{q}$ is disjoint with the ball $\mathbb{B}_{R^{0}}$; then $u^{q} \in \mathcal{H}$ by (1.11) and the definition of the space $\stackrel{\circ}{H}_{\text {per }}^{1}(\Pi ; \gamma)^{3}$ (see the comment on formula (2.6)).

Since the product $\mathcal{X}_{q}$ of the cutoff functions on the right in (2.46) is equal to 1 for $y_{1} \in\left(2^{q}+1,2^{q+1}-1\right)$, the second condition in (1.10) allows us to write

$$
\begin{aligned}
\left(\rho u^{q}, u^{q}\right)_{\Omega} & \geq 2^{-q}\left(2^{q+1}-2^{q}-2\right)\left(\left\|\mathbf{u} ; L_{2}(\Pi)\right\|^{2}-c \exp \left(-\delta_{0} 2^{q}\right)\right) \\
& \geq c_{1}-c_{0} 2^{-q} \geq c_{1} / 2
\end{aligned}
$$

Here $c_{0}$ and $c_{1}$ are positive constants, and the index $q$ is assumed to be large.

Similarly,

$$
\left(\rho u^{q}, u^{q}\right)_{\Omega} \leq c_{2}, \quad\left(A D\left(\nabla_{x}\right) u^{q}, D\left(\nabla_{x}\right) u^{q}\right)_{\Omega} \leq c_{3} .
$$

Hence, the sequence (2.46) is bounded and uniformly separated away from zero in the norm of the space $\mathcal{H}$, and it converges to zero weakly in $\mathcal{H}$, because the supports of $u^{q}$ and $u^{p}$ are disjoint for $q \neq p$. Thus, it remains to verify the third fundamental property of a Weyl sequence (see, e.g., [11, §9.1]), namely,

$$
\left\|\mathcal{K} u^{q}-\mu u^{q} ; \mathcal{H}\right\| \rightarrow 0, \quad q \rightarrow+\infty .
$$

We have

$$
\begin{aligned}
\left\|\mathcal{K} u^{q}-\mu u^{q} ; \mathcal{H}\right\|= & \sup \left\{\left|\left\langle\mathcal{K} u^{q}-\mu u^{q}, w\right\rangle\right|: w \in \mathcal{H},\|w ; \mathcal{H}\|=1\right\}, \\
\left\langle\mathcal{K} u^{q}-\mu u^{q}, w\right\rangle= & (1+\lambda)^{-1}\left(\left(A D\left(\nabla_{x}\right) u^{q}, D\left(\nabla_{x}\right) w\right)_{\Omega}-\lambda\left(\rho u^{q}, w\right)_{\Omega}\right) \\
= & (1+\lambda)^{-1}\left(\left(A^{0} D\left(\nabla_{x}\right) u^{q}, D\left(\nabla_{x}\right) w\right)_{\Omega_{0}}-\lambda \rho_{0}\left(u^{q}, w\right)_{\Omega_{0}}\right) \\
& +O\left(\exp \left(-\delta_{0} 2^{q}\right)\right) .
\end{aligned}
$$

Here we have used formulas (1.10) and (1.12)-(1.14). Putting $v(x)=\mathcal{X}_{q}\left(y_{1}\right) w(x)$, in the last-written inner product in (2.48) we transfer the cutoff function $\mathcal{X}_{q}$, and also the factor $\exp \left(i \eta_{0} y_{1}\right)$ from the vector-valued function $\mathbf{u}$ (see (2.46)) to the test function $w$. Since the support of the commutator $D\left(\nabla_{x} \mathcal{X}_{q}\right)$ lies in the closure of only two prisms $\Pi_{2^{q}}$ 
and $\Pi_{2^{q+1}-1}$, we obtain

$$
\begin{aligned}
& \left(A^{0} D\left(\nabla_{x}\right) u^{q}, D\left(\nabla_{x}\right) w\right)_{\Omega_{0}}-\lambda \rho_{0}\left(u^{q}, w\right)_{\Omega_{0}} \\
& =2^{-q / 2}\left(\left(A^{0} D\left(\partial_{1}+i \eta_{0}, \partial_{2}, \partial_{3}\right) \mathbf{u}, D\left(\partial_{1}+i \eta, \partial_{2}, \partial_{3}\right) \exp \left(i \eta_{0} y_{1}\right) v\right)_{\Omega_{0}}\right. \\
& \left.-\lambda \rho_{0}\left(\mathbf{u}, \exp \left(i \eta_{0} y_{1}\right) v\right)_{\Omega_{0}}+O(1)\right) \\
& =2^{-q / 2}\left(\left(A^{0} D\left(\partial_{1}+i \eta_{0}, \partial_{2}, \partial_{3}\right) \mathbf{u}, D\left(\partial_{1}+i \eta_{0}, \partial_{2}, \partial_{3}\right) \mathbf{v}\right)_{\Pi}\right. \\
& \left.-\lambda \rho_{0}(\mathbf{u}, \mathbf{v})_{\Pi}+O(1)\right) \\
& =O\left(2^{-q / 2}\right) \text {. }
\end{aligned}
$$

Here, we applied the definition (2.2) of the Gelfand image $\mathbf{v}=\widehat{v}$, and then identity (2.6) for the spectral pair $\left\{\eta_{0}, \mathbf{u}\right\}$ of the pencil $\mathfrak{a}(\cdot ; \lambda)$. Relations (2.48) and (2.49) imply the convergence (2.47).

If a point $\eta_{0}$ of the continuous spectrum of the pencil $\eta \mapsto \mathfrak{a}(\eta ; \lambda)$ lies on $[0,2 \pi)$, then, by Theorem 2.1, the pencil $\zeta \mapsto \mathfrak{A}(\zeta ; \eta, \lambda)$ has a real eigenvalue $\zeta_{0}$. Let $U$ denote an eigenfunction corresponding to $\zeta_{0}$. The construction of elements of an appropriate singular Weyl sequence differs little from that used earlier in (2.46):

$$
u^{q}(x)=2^{-q} \mathcal{X}_{q}\left(y_{1}\right) \mathcal{X}_{q}(z) \exp \left(i \eta_{0} y_{1}\right) \exp \left(i \zeta_{0} z\right) U(y) .
$$

The function $z \mapsto \mathcal{X}_{q}(z)$, cutting off in the vertical direction, is added because the solution $\mathcal{U}(x)=\exp \left(i \zeta_{0} z\right) U(y)$ of the system of differential equations

$$
L\left(\nabla_{y}, \partial_{z} ; i \eta\right) \mathcal{U}(x)=\lambda \rho_{0} \mathcal{U}(x), \quad x \in \Pi^{\infty}
$$

(see the definition of the differential operator in the first line of (2.11)) satisfies the Dirichlet conditions (1.6) on the faces $\{ \pm 1 / 2\} \times(-l / 2, l / 2) \times \mathbb{R}$ of the prism and the periodicity conditions on the other two faces, i.e., possesses the required properties of the function $\mathbf{u}$ in (2.46), but does not belong to the Sobolev space $H^{1}(\Pi)^{3}$. The factor $2^{-q}$ is responsible for the preservation of inequality (2.5) (of course, without its middle part). The other calculations and arguments need no essential changes.

By Theorem 2.2 and the remarks about the operator $m(\eta)$ made before the formulation of that theorem, the segment $\left[0, \mu_{+}^{0}\right]$ (the ray $\left[\lambda_{+}^{0},+\infty\right)$ ) is occupied by the continuous spectrum of the operator $\mathcal{K}$ (the continuous spectrum of problem (1.12)). The absolute thresholds

$$
\mu_{\dagger}^{0}=\left(1+\lambda_{\dagger}\right)^{-1}, \quad \lambda_{\dagger}^{0}=\left(\rho^{0}\right)^{-1} \min \left\{\Lambda_{\min }(\eta): \eta \in[0,2 \pi)\right\}
$$

are related to the continuous spectrum of the pencil (2.7) (i.e., to the loss of the Fredholm property by the operator of the model problem (2.6) on the section $\Theta$ of the periodicity cell $\Pi$ ), and are only determined by the limiting rigidity matrix $A^{0}$ and by the size $l$ (see (1.10) and (1.2), respectively), via the eigenvalues (2.27) of the pencil (2.12) (see formula (2.27)).

Besides the thresholds (2.50), partial thresholds may arise:

$$
\mu_{\dagger}^{(n)}=\left(1+\lambda_{\dagger}^{(n)}\right)^{-1}, \quad \lambda_{\dagger}^{(n)}=\rho_{0}^{-1} \Lambda_{\min }^{(n)},
$$

where $n=1, \ldots, N_{\max }, N_{\max }=\max \{N(\eta): \eta \in[0,2 \pi)\}$,

$$
\Lambda_{\min }^{(n)}=\min \left\{\Lambda^{(n)}(\eta): \eta \in[0,2 \pi)\right\},
$$

and the number $\Lambda_{\max }^{(n)}$ is defined similarly. Here we have used the eigenvalues of the operators $m(\eta)$, and if $\Lambda^{(n)}(\eta)$ is absent on the list (2.36), then we put $\Lambda^{(n)}(\eta)=+\infty$. The partial thresholds (2.51) are generated by the discrete spectrum (2.36) of $m(\eta)$. By 
Theorem 2.2 and the continuous dependence of $\Lambda^{(n)}(\eta) \in\left[0, \Lambda_{\min }(\eta)\right]$ on $\eta$, each of the thresholds (2.51) is an end, upper or lower (respectively), of the segments

$$
\Delta^{(n)}=\left[\mu_{\#}^{(n)}, \mu_{\dagger}^{(n)}\right] \quad \text { and }\left[\lambda_{\dagger}^{(n)}, \lambda_{\#}^{(n)}\right]
$$

forming the continuous spectra of the operator $\mathcal{K}$ and problem (1.12), and

$$
\lambda_{\#}^{(n)}=\rho_{0}^{-1} \Lambda_{\max }^{(n)}, \quad \mu_{\#}^{(n)}=\left(1+\lambda_{\#}^{(n)}\right)^{-1} .
$$

Since the continuous spectrum of problem (1.12) is a union of finite and half-infinite segments (in particular, $\left[\lambda_{\#}^{0},+\infty\right)$ ), this spectrum can admit gaps, i.e., intervals containing discrete spectrum points only. An example of such a gap is presented in the next section.

5. On the exponential decay of solutions at infinity. By what was said about the eigenvalues of the pencil (2.12), in particular since they belong to the set (2.14), the absence of points of the spectrum on the real axis means that there are no such points also in a strip $\{\zeta \in \mathbb{C}: \operatorname{Im} \zeta \in(-\delta(\lambda), 0]\}$ for some $\delta(\lambda)>0$ and all $\eta \in[0,2 \pi)$. It is well known (see [12] and also, e.g., [16, Chapter 3]) that this property of the spectrum ensures the exponential decay of solutions of problem (2.15) with appropriate right-hand sides. Now we formulate the result, postponing comments till Proposition 2.2, where a similar statement will be established for problem (2.38).

Proposition 2.1. Suppose that, for some $\lambda \geq 0$ and $\eta \in[0,2 \pi)$, the real axis is free from the spectrum of the pencil $\zeta \mapsto \mathfrak{A}(\zeta ; \lambda, \eta)$. Also, suppose that a functional $\mathbf{f} \in$ $\left(\stackrel{\circ}{H}_{\text {per }}^{1}(\Pi ; \gamma)^{3}\right)^{*}$ satisfies

$$
|\mathbf{f}(\exp (-\delta z) \mathbf{v})| \leq \mathbf{c}_{\delta}(\mathbf{f})\left\|\mathbf{v} ; H^{1}(\Pi)\right\|, \quad v \in \stackrel{\circ}{H}_{\mathrm{per}}^{1}(\Pi ; \gamma)^{3} .
$$

There exists a number $\delta(\lambda, \eta)>0$ such that if $\delta \in(0, \delta(\lambda, \eta))$, then for the solution $\mathbf{u} \in \stackrel{\circ}{H}_{\text {per }}^{1}(\Pi ; \gamma)^{3}$ of problem (2.15) we have $\exp (-\delta z) \mathbf{u} \in \stackrel{\circ}{H}_{\text {per }}^{1}(\Pi ; \gamma)^{3}$ and

$$
\left\|\exp (-\delta z) \mathbf{u} ; H^{1}(\Pi)\right\| \leq c_{\delta}\left(\mathbf{c}_{\delta}(\mathbf{f})+\left\|\mathbf{f} ;\left(\stackrel{\circ}{H}_{\mathrm{per}}^{1}(\Pi ; \gamma)^{3}\right)^{*}\right\|+\left\|\mathbf{u} ; H^{1}(\Pi)\right\|\right) .
$$

The constant $c_{\delta}$ depends on $\delta$, but not on $\mathbf{u}$ and $\mathbf{f}$, and $\mathbf{c}_{\delta}(\mathbf{f})$ is the coefficient occurring on the right-hand side in (2.53).

In particular, Proposition 2.1 implies that the eigenfunctions corresponding to the eigenvalues (2.36) decay exponentially as $z \rightarrow-\infty$.

Remark 2.4. Independently of the presence or absence of the spectrum of the pencil $\mathfrak{A}$ on the line $\mathbb{R} \subset \mathbb{C}$, the kernel of the operator of problem (2.15), viewed as the map (2.7), turns out to be finite-dimensional. Indeed, we can always find $\delta>0$ such that the line $\mathbb{R}+i \delta=\{\zeta \in \mathbb{C}: \operatorname{Im} \zeta=\delta\}$ is free from the spectrum of the pencil. By the results of [12] (see also [16, Chapter 3] and the proof of Theorem 2.1), the operator of problem (2.15), acting from the space

$$
\stackrel{\circ}{W}_{\delta, \text { per }}^{1}(\Pi ; \gamma)^{3}:=\left\{\mathbf{u} \in H_{\mathrm{loc}}^{1}(\bar{\Pi})^{3}: \exp (\delta \zeta) \mathbf{u} \in \stackrel{\circ}{H}_{\mathrm{per}}^{1}(\Pi ; \gamma)^{3}\right\}
$$

of exponentially decaying vector-valued functions to the space

$$
\left(\stackrel{\circ}{W}_{-\delta, \operatorname{per}}^{1}(\Pi ; \gamma)^{3}\right)^{*}:=\left\{\mathbf{f}:|\mathbf{f}(\exp (\delta z) \mathbf{v})| \leq c\left\|\mathbf{v} ; H^{1}(\Pi)\right\|, \mathbf{v} \in \stackrel{\circ}{H}_{\text {per }}^{1}(\Pi ; \gamma)^{3}\right\},
$$

of functionals, is Fredholm, so that its kernel is finite-dimensional. It remains to observe that, since the space (2.54) is wider than $\stackrel{\circ}{\mathrm{per}}^{1}(\Pi ; \gamma)^{3}$, the kernel could only become larger when we pass to functions that grow at infinity. Consequently, the kernel of the operator defined on $\stackrel{\circ}{H}_{\text {per }}^{1}(\Pi ; \gamma)^{3}$ is also finite-dimensional. 
We turn to problem (1.4)-(1.6), or (1.12). We equip the space

$$
\mathcal{W}(\delta)=\left\{u \in H_{\mathrm{loc}}^{1}(\bar{\Omega})^{3}: \exp \left(\delta\left(\left|y_{1}\right|-z\right)\right) u \in \mathcal{H}\right\}
$$

with the natural norm $\|u ; \mathcal{W}(\delta)\|=\left\|\exp \left(\delta\left(\left|y_{1}\right|-z\right)\right) u ; \mathcal{H}\right\|$. Clearly, $\mathcal{W}(0)=\mathcal{H}$. If $\delta>0$, then the vector-valued functions in the space (2.56) decay exponentially at infinity, but if $\delta<0$, then certain growth is allowed.

Let $\mathcal{W}(\delta)^{*}$ be the dual space. Since $\left(C_{c}^{\infty}(\bar{\Omega}) \cap \mathcal{H}\right)^{3}$ is dense in $\mathcal{W}(\delta)$, the integral identity (2.38) remains meaningful for any $u \in \mathcal{W}(\delta), f \in \mathcal{W}(-\delta)^{*}$, and $v \in \mathcal{W}(-\delta)$. Thereby, this identity gives rise to an operator

$$
\mathfrak{Q}_{\delta}: \mathcal{W}(\delta) \rightarrow \mathcal{W}(-\delta)^{*}
$$

which coincides with the operator considered before for $\delta=0$.

If the half-interval $[0,2 \pi)$ is free from the spectrum of the pencil (2.7), then, for some $\delta(\lambda)>0$, the rectangle

$$
\{\eta \in \mathbb{C}: \operatorname{Re} \eta \in[0,2 \pi), \operatorname{Im} \eta \in(-\delta(\lambda), \delta(\lambda)\} .
$$

possesses the same property. Let $\delta \in(-\delta(\lambda), \delta(\lambda))$. We extend the Gelfand transformation (2.2) to complex values of $\eta$, and in the inverse transformation (2.3) we shift integration to the half-interval $\{\eta \in \mathbb{C}: \operatorname{Re} \eta \in[0,2 \pi), \operatorname{Im} \eta=\delta\}$ (cf. [33] and [16, §3.4]).

Consider the nonhomogeneous model problem (2.1) and introduce the space $\mathcal{W}_{0}(\delta)$ by formula (2.56) with $\mathcal{H}_{0}$ in place of $\mathcal{H}$. As the right-hand side of a problem on the periodic set $\Omega_{0}$, we take a functional $f^{0} \in \mathcal{W}_{0}\left(-\delta_{1}\right)^{*}$, where $\delta_{1} \in(0, \delta(\lambda))$. Since $v \mapsto$ $f^{0}\left(\exp \left( \pm \delta y_{1}\right) v\right)$ is a continuous functional belonging to the dual space $\mathcal{H}^{*}$ for all $\delta \in$ $\left[0, \delta_{1}\right]$, and $\partial_{\eta} \widehat{v}$ is the image of the function $x \mapsto-i y_{1} v(x)$, the Gelfand transformation yields an abstract $2 \pi$-periodic function holomorphic in the strip and continuous up to the boundary of the half-strip. The operator of problem (2.15), viewed as a map

$$
\stackrel{\circ}{W}_{\delta_{1}, \operatorname{per}}^{1}(\Pi ; \gamma)^{3} \rightarrow\left(\stackrel{\circ}{W}_{-\delta_{1}, \mathrm{per}}^{1}(\Pi ; \gamma)^{3}\right)^{*}
$$

(see (2.54) and (2.55)), has a resolvent $\mathfrak{R}_{\delta}(\eta)$ holomorphic in the strip $\{\eta:|\operatorname{Im} \eta|<\delta(\lambda)\}$ and $2 \pi$-periodic along the real axis. As a result, the inverse Gelfand transformation determines a family, parametrized by $\delta \in\left[-\delta_{1}, \delta_{1}\right]$, of solutions

$$
v^{\delta}(x)=\frac{1}{\sqrt{2 \pi}} \int_{i \delta}^{2 \pi+i \delta} \exp \left(i \eta y_{1}\right) \mathfrak{R}_{\delta}(\eta) \widehat{f}^{0}(\cdot ; \eta) d \eta
$$

of the model problem on the periodic set $\Omega_{0}$. These solutions do not differ from one another, because the difference of two solutions is representable as a contour integral along the boundary of the rectangle $v^{\delta_{1}}-v^{\delta_{2}}$, and the integrals along its lateral sides cancel, because of the opposite directions of integration and the $2 \pi$-periodicity of the integrand with respect to the variable $\operatorname{Re} \eta$. Thus, for the solution $v^{0} \in \mathcal{H}_{0}$ we have $\exp \left(\delta y_{1}\right) \exp (-\delta z) v^{0} \in \mathcal{H}$ for all $\delta \in\left[-\delta_{1}, \delta_{1}\right]$, whence $v^{0} \in \mathcal{W}_{0}\left(\delta_{1}\right)$. Also,

$$
\left\|v^{0} ; \mathcal{W}_{0}\left(\delta_{1}\right)\right\| \leq c\left\|f^{0} ; \mathcal{W}_{0}\left(-\delta_{1}\right)^{*}\right\| .
$$

Now, let $u \in \mathcal{H}$ be a solution of problem (2.38) with a right-hand side $f \in \mathcal{W}(-\delta)^{*}$ for some $\delta>0$. We multiply a test function $v$ by a smooth cutoff function $X$ equal to 0 for $|x|<R^{0}$ and to 1 for $|x|>2 R^{0}$ and transfer the following forms to the right:

$$
\begin{aligned}
& \left(\left(A-A^{0}\right) D\left(\nabla_{x}\right) u, D\left(\nabla_{x}\right)(X v)\right)_{\Omega}, \quad \lambda\left(\left(\rho-\rho_{0}\right) u, X v\right)_{\Omega}, \\
& \left(A^{0} D\left(\nabla_{x}\right) u, D\left(\nabla_{x} X\right) v\right)_{\Omega}, \quad-\left(A^{0} D\left(\nabla_{x} X\right) u, D\left(\nabla_{x}\right) v\right)_{\Omega} .
\end{aligned}
$$

In the first pair we have an additional weight factor $\exp \left(-\delta_{0}|x|\right)$, occurring in the stabilization condition (1.10), and in the second pair integration is over a compact set. Thus, 
the product $v^{0}=X v$ turns out to be a solution of the model problem in $\Omega_{0}$ with the right-hand side $f^{0} \in \mathcal{W}_{0}\left(-\delta_{1}\right)^{*}$, where $\delta_{1}=\min \left\{\delta, \delta_{0}\right\}$. So, the above arguments, which followed the lines of [33] (see also [16, §3.4]), imply the next statement.

Proposition 2.2. Suppose $\delta \in\left(0, \delta_{0}\right]($ see (1.10) $)$ and $f \in \mathcal{W}(-\delta)^{*}$. Then the solution $u \in \mathcal{H}$ of problem (2.38) belongs to $\mathcal{W}(\delta)$ and satisfies the estimate $\|u ; \mathcal{W}(\delta)\| \leq$ $c_{\delta}\left\|f ; \mathcal{W}(-\delta)^{*}\right\|$.

In particular, this shows that the eigenfunction $u$ corresponding to a point $\mu$ of the discrete spectrum of $\mathcal{K}$ decays exponentially at infinity.

The verification of Proposition 2.1 repeats, with simplifications, the above proof of Proposition 2.2.

Remark 2.5. In contrast to the kernel of the operator $m(\eta)$, mentioned in Remark 2.4, the kernel of the operator $\mathcal{K}-\mu$ may fail to be finite-dimensional. Here is a simplest example. Let $\Omega=\Omega_{0}$, let $A=A^{0}$, and suppose that for all $\eta$ on a segment $\left[\eta_{1}, \eta_{2}\right] \subset[0,2 \pi)$ of positive length the operator $m(\eta)$ has an eigenvalue $\Lambda=\rho_{0} \lambda$. The corresponding eigenfunction

$$
\mathbf{u}(\cdot ; \eta) \in \stackrel{\circ}{H}_{p e r}^{1}(; \gamma)^{3}
$$

decays exponentially at infinity by Proposition 2.1. Consequently, for any density $\varphi \in$ $C_{c}^{\infty}\left(\eta_{1}, \eta_{2}\right)$ the formula

$$
u^{\varphi}(x)=\frac{1}{\sqrt{2 \pi}} \int_{0}^{2 \pi} \exp \left(i \eta y_{1}\right) \mathbf{u}\left(y_{1}-\left[y_{1}\right], y_{2}, z ; \eta\right) d \eta
$$

gives us a solution of the homogeneous problem, and this solution belongs to the space $\mathcal{H}=\mathcal{H}_{0}$.

\section{§3. SPECIFIC FORMS}

1. The Korn inequality. Let $\Xi$ be a 3-dimensional elastic body with Lipschitz boundary $\Xi$ and compact closure $\Xi$. A part $\Gamma$ of the surface of $\Xi$ is clamped rigidly; i.e., Dirichlet conditions of the form (1.6) are preassigned on $\Gamma$. Various methods for the proof of the Korn inequality

$$
\left\|u ; H^{1}(\Xi)\right\|^{2} \leq c_{\Xi}\left\|D\left(\nabla_{x}\right) u ; L_{2}(\Xi)\right\|^{2}, \quad u \in \stackrel{\circ}{H}^{1}(\Xi ; \Gamma)^{3},
$$

which was already used in the proofs of Lemmas 2.1 and 2.2, can be found in 39]-37]. Largely, the dependence of the Korn constant $c_{\Xi}$ on the domain $\Xi$ is not known; in [37] it was proved only that if a body $\Xi$ is star-shaped relative to the ball $\mathbb{B}_{R}$, then the constant $c$ in the inequality

$$
\left\|u ; H^{1}(\Xi)\right\|^{2} \leq c\left(\left\|D\left(\nabla_{x}\right) u ; L_{2}(\Xi)\right\|^{2}+\left\|u ; L_{2}\left(\mathbb{B}_{R}\right)\right\|^{2}\right), \quad u \in H^{1}(\Xi)^{3},
$$

can be taken depending only on the ratio of the diameters $D$ and $2 R>0$ of the domains $\Xi$ and $\mathbb{B}_{R}$.

In the case of thin elastic bodies (plates and rods), to make the Korn inequality asymptotically sharp relative to the small geometric parameter $h>0$, we should consider anisotropic and weighted Sobolev norms, (3.1) and (3.2), on the left-hand sides in 42 and [43] (see also the book [6] and the survey [44, $\S 2]$ ). Here we present such an inequality, anisotropic and weighted, see [43, 45, for a rod $G_{h}=\omega_{h} \times(0,1)$ with cross section 
$\omega_{h}=\left\{y: h^{-1} y \in \omega\right\}$, where $\omega$ is a domain in $\mathbb{R}^{2}$ bounded by a simple closed contour $\partial \omega:$

$$
\begin{aligned}
\mathbf{|} w ; G_{h} \mathbf{l}^{2}:= & \left\|\frac{\partial w_{p}}{\partial y_{p}} ; L_{2}\left(G_{h}\right)\right\|^{2}+\left\|\frac{\partial w_{3}}{\partial z} ; L_{2}\left(G_{h}\right)\right\|^{2} \\
& +h^{2}\left(\left\|\rho_{h}^{-1} \frac{\partial w_{p}}{\partial z} ; L_{2}\left(G_{h}\right)\right\|^{2}+\left\|\rho_{h}^{-1} \frac{\partial w_{3}}{\partial y_{p}} ; L_{2}\left(G_{h}\right)\right\|^{2}\right. \\
& \left.\quad+\left\|\rho_{h}^{-1} \frac{\partial w_{1}}{\partial y_{2}} ; L_{2}\left(G_{h}\right)\right\|^{2}+\left\|\rho_{h}^{-1} \frac{\partial w_{2}}{\partial y_{1}} ; L_{2}\left(G_{h}\right)\right\|^{2}\right) \\
& +\left\|\rho_{h}^{-1} w_{3} ; L_{2}\left(G_{h}\right)\right\|^{2}+h^{2}\left\|\rho_{h}^{-2} w_{p} ; L_{2}\left(G_{h}\right)\right\|^{2} \\
\leq & c\left\|D\left(\nabla_{x}\right) w ; L_{2}\left(G_{h}\right)\right\|^{2} .
\end{aligned}
$$

Here $p=1,2$, and $\rho_{h}(x)=z+h$ is a weight factor equal to $O(1)$ far from the clamped end $\omega_{h}(0)=\omega_{h} \times\{0\}$ of the rod (for $z>c>0$ ), and equal to $O(h)$ near that end (for $z \leq C h$ ). In [45, 46] it was shown that for many purposes inequality (3.3) is insufficient, and that an additional relation of the form

$$
\left\|\rho_{h}^{-1}\left(w_{p}-\bar{w}_{p}\right) ; L_{2}\left(G_{h}\right)\right\|^{2} \leq c\left\|D\left(\nabla_{x}\right) w ; L_{2}\left(G_{h}\right)\right\|^{2}
$$

is required. In (3.4), the quantities

$$
\bar{w}_{p}(z)=\left(\operatorname{meas}_{2} \omega_{h}\right)^{-1} \int_{\omega_{h}} w_{p}(y, z) d y
$$

are the mean displacements. Of course, the constants $c$ in (3.3) and (3.4) are independent of $h \in(0,1]$ and $w \in \stackrel{\circ}{H}^{1}\left(G_{h} ; \omega_{h}(0)\right)^{3}$. The proof of inequalities (3.3) and (3.4) can be found in [6, Chapter 3] and [44, §2].

2. Cushion with fringe. Suppose that the periodicity cell has the form

$$
\Pi=\Pi(1 / 6) \cup G_{h} \cup B,
$$

where $\Pi(H)$ is a half-infinite prism (1.2), $B=\left\{x:|y|^{2}+|z-5 / 6|^{2}<1 / 4\right\}$ is the ball of radius $1 / 2$ centered at $x=(0,0,5 / 6)$, and $G_{h}$ is the rod mentioned in the preceding subsection. The number $h_{0}$ will be chosen so that $G_{h} \subset \Pi(\infty)=\left\{x=(y, z):\left|y_{p}\right|<\right.$ $\left.l_{p} / 2, z \in \mathbb{R}\right\}$ for $h \in\left(0, h_{0}\right]$. We assume that the disk $\{y:|y|<1\}$ lies inside the reduced $(h=1)$ section $\omega$; then the rod $G_{h}$ includes the cylinder $\varpi_{h}=\{x:|y|<h,|z-3 / 4|<$ $1 / 4\}$. For the role of the clamped surfaces we take the half-planes $\Sigma^{ \pm}=\Sigma_{0}^{ \pm}=\left\{x: y_{2}=\right.$ $\pm l / 2, z<0\}$.

Plane pictures of the periodicity cell and the cushion with fringe $\Omega$ are presented in Figures 4 and 6 , respectively; to form the periodic set $\Omega_{0}$ (Figure 5 ) one needs to restore the two elements of the fringe that are defective in Figure 6.

Now we deduce an asymptotically sharp, weighted and anisotropic, Korn inequality for the cell (3.6); we use the method described in [19] (see also [44, §3]). Let $u \in$ $\stackrel{\circ}{H}^{1}(\Pi ; \Sigma \cap \partial \Pi)^{3}$. Repeating the proof of Lemma 2.1. we arrive at the relation

$$
\left\|u ; H^{1}(\Pi(1 / 6))\right\|^{2} \leq c\left\|D\left(\nabla_{x}\right) w ; L_{2}(\Pi)\right\|^{2} .
$$

We employ the corollary

$$
\left\||x|^{-1} u ; L_{2}(\Theta \times(-1 / 6,1 / 6))\right\|^{2} \leq c\left\|u ; H^{1}(\Theta \times(-1 / 6,1 / 6))\right\|^{2}
$$

to the one-dimensional Hardy inequality

$$
\int_{0}^{\infty}|W(r)|^{2} d r \leq 4 \int_{0}^{\infty} r^{2}\left|\frac{d W}{d r}(r)\right|^{2} d r, \quad W \in C_{c}^{1}[0,+\infty),
$$


involving the radial variable $r=|y|$ and integrated over the angular variables of the spherical system of coordinates. As a result, for the field $w(x)=X\left(h^{-1} z\right) u(x)$ on the $\operatorname{rod} G_{h}$ we obtain the estimate

$$
\begin{aligned}
& \left\|D\left(\nabla_{x}\right) w ; L_{2}\left(G_{h}\right)\right\|^{2} \leq 2\left\|D\left(\nabla_{x}\right) u ; L_{2}\left(G_{h}\right)\right\|+2\left\|D\left(\partial_{z} X\right) u ; L_{2}\left(G_{h}\right)\right\|^{2} \\
& \quad \leq 2\left\|D\left(\nabla_{x}\right) u ; L_{2}\left(G_{h}\right)\right\|^{2}+c h^{-2}\left\|u ; L_{2}\left(\omega_{h} \times(0, h)\right)\right\|^{2} \\
& \quad \leq 2\left\|D\left(\nabla_{x}\right) u ; L_{2}\left(G_{h}\right)\right\|^{2}+c\left\||x|^{-1} ; L_{2}\left(\omega_{h} \times(0, h)\right)\right\|^{2} \\
& \quad \leq\left\|D\left(\nabla_{x}\right) u ; L_{2}\left(\Pi(1 / 6) \cup G_{h}\right)\right\|^{2} .
\end{aligned}
$$

Here $X$ is the same cutoff function as in (2.46). Since $w(y, 0)=0$ because of cutting off, we have inequalities (3.3) and (3.4) at our disposal. Observe that, first, in the middle of the calculations (3.10) we used the fact that $r<c h$ on the cylinder $\omega_{h} \times(0, h)$, and second, to deduce formula (3.8) from the Hardy inequality (3.9) we should put $W(r)=\chi(r) u(x)$, where $\chi$ is an appropriate cutoff function.

We represent the restriction of the vector-valued function $u$ to a ball $B$ in the form

$$
u(x)=u^{\perp}(x)+d(y, z-3 / 4) a, \quad \int_{B} d\left(y, z-\frac{3}{4}\right)^{\top} u^{\perp}(x)=0 \in \mathbb{R}^{6},
$$

where $d(x) a$ is the rigid displacement (i.e., $a$ is a column in $\mathbb{R}^{6}$ and $d$ is a linear matrixvalued function of size $3 \times 6$, similar to the matrix $D(x)^{\top}$ in formula (1.8)),

$$
d(x)=\left(\begin{array}{cccccc}
1 & 0 & 0 & 0 & 2^{-1 / 2} x_{3} & -2^{-1 / 2} x_{2} \\
0 & 1 & 0 & -2^{-1 / 2} x_{3} & 0 & 2^{-1 / 2} x_{1} \\
0 & 0 & 1 & 2^{-1 / 2} x_{2} & -2^{-1 / 2} x_{1} & 0
\end{array}\right) .
$$

Due to the orthogonality conditions in (3.11), the following version of the Korn inequality is valid (see [41, 37] and, e.g., 6, §2.2] and [44, §2]):

$$
\left\|u^{\perp} ; H^{1}(B)\right\|^{2} \leq c\left\|D\left(\nabla_{x}\right) u^{\perp} ; L_{2}(B)\right\|^{2}=c\left\|D\left(\nabla_{x}\right) u ; L_{2}(B)\right\|^{2} .
$$

The last identity is ensured by the relation $D\left(\nabla_{x}\right) d(x)=0$ implied by (1.8) and (3.12) (strains vanish at the rigid displacements).

It remains to handle the column $a$. Formulas (3.11) show that

$$
a=\left\{\int_{\varpi_{h}} d\left(y, z-\frac{3}{4}\right)^{\top} d\left(y, z-\frac{3}{4}\right) d x\right\}^{-1} \int_{\varpi_{h}} d\left(y, z-\frac{3}{4}\right)^{\top}\left(u(x)-u^{\perp}(x)\right) d x
$$

and the Gram matrix occurring in braces becomes diagonal because the point $(0,0,3 / 4)$ lies in the center of the circular cylinder $\varpi_{h}$; namely, this Gram matrix looks like this:

$$
\operatorname{diag}\left\{\frac{\pi}{2} h^{2}, \frac{\pi}{2} h^{2}, \frac{\pi}{2} h^{2}, \frac{\pi}{16} h^{2}\left(\frac{1}{12}+h^{2}\right), \frac{\pi}{16} h^{2}\left(\frac{1}{12}+h^{2}\right), \frac{\pi}{8} h^{4}\right\} .
$$

The first three positions are occupied by the volume of the cylinder $\varpi_{h}$, and the last three by its moments of inertia.

By (3.14), we have

$$
\left|a_{q}\right|^{2} \leq \operatorname{ch}^{-4} \operatorname{meas}_{3} \varpi_{h} \int_{\varpi_{A}}\left(\left|u_{q}(x)\right|^{2}+\left|u_{q}^{\perp}(x)\right|^{2}\right) d x, \quad q=1,2,3 .
$$

First, let $q=3$. We observe that meas $_{3} \varpi_{h}=O\left(h^{2}\right)$ and $u_{q}=w_{q}$ on $\varpi_{h}$ and estimate the integral in (3.15) with the help of (3.3) and (3.13), obtaining

$$
\left|a_{3}\right|^{2} \leq c h^{-2}\left\|D\left(\nabla_{x}\right) u ; L_{2}(\Pi)\right\|^{2} .
$$


For $q=1,2$, the factor $h^{-1}$ arises in the majorant for the norm $\left\|w_{q} ; L_{2}\left(\varpi_{h}\right)\right\|$ because of the anisotropic structure of the Korn inequality (3.3). As a result, we obtain

$$
\begin{aligned}
\left|a_{q}\right|^{2} & \leq c h^{-2}\left(\left\|w_{q} ; L_{2}\left(\varpi_{h}\right)\right\|^{2}+\left\|u_{q}^{\perp} ; L_{2}\left(\varpi_{h}\right)\right\|^{2}\right) \\
& \leq c h^{-2}\left(h^{-2}\left\|D\left(\nabla_{x}\right) u ; L_{2}\left(\Pi(1 / 6) \cup G_{h}\right)\right\|^{2}+\left\|D\left(\nabla_{x}\right) u ; L_{2}(B)\right\|^{2}\right) \\
& \leq c h^{-4}\left\|D\left(\nabla_{x}\right) u ; L_{2}(\Pi)\right\|^{2} .
\end{aligned}
$$

A similar inequality is fulfilled for $q=4,5$ :

$$
\begin{aligned}
\left|a_{6-p}\right|^{2} & \leq c h^{-2}\left(\left\|w_{p} ; L_{2}\left(\varpi_{h}\right)\right\|^{2}+h^{2}\left\|w_{3} ; L_{2}\left(\varpi_{h}\right)\right\|^{2}+\left\|u^{\perp} ; L_{2}\left(\varpi_{h}\right)\right\|^{2}\right) \\
& \leq c h^{-4}\left\|D\left(\nabla_{x}\right) u ; L_{2}(\Pi)\right\|^{2}, \quad p=1,2 .
\end{aligned}
$$

Finally,

$$
\begin{aligned}
\left|a_{6}\right|^{2} \leq & c h^{-8}\left(\left|\int_{\varpi_{h}}\left(y_{1} w_{2}(x)-y_{2} w_{1}(x)\right) d x\right|^{2}+\left|\int_{\varpi_{h}}\left(y_{1} u_{2}^{\perp}(x)-y_{2} u_{1}^{\perp}(x)\right) d x\right|^{2}\right) \\
& \leq c h^{-8}\left(\left|\int_{\varpi_{h}}\left(y_{1}\left(w_{2}(x)-\overline{w_{2}}(z)\right)-y_{2}\left(w_{1}(x)-\overline{w_{1}}(z)\right)\right) d x\right|^{2}\right. \\
& \left.\quad+h^{2} \operatorname{meas}_{2} \varpi_{h} \int_{\varpi_{h}}\left|u^{\perp}(x)(x)\right|^{2} d x\right) \\
& \leq c h^{-4}\left(\left\|D\left(\nabla_{x}\right) w ; L_{2}\left(G_{h}\right)\right\|^{2}+\left\|D\left(\nabla_{x}\right) u^{\perp} ; L_{2}(B)\right\|^{2}\right) \\
& \leq c h^{-4}\left\|D\left(\nabla_{x}\right) u ; L_{2}(\Pi)\right\|^{2} .
\end{aligned}
$$

Note that, instead of the Korn inequality (3.3), here we have used inequality (3.4) involving the mean values (3.5) of the displacements $w_{p}$ on a section of the rod $G_{h}$, and that the substitutions $w_{p} \mapsto w_{p}-\bar{w}_{p}$ in the first integral in the chain (3.19) are possible, because the integral of $y_{3-p}$ over a circular section of the cylinder $\varpi_{h}$ vanishes.

Collecting relations (3.7), (3.15)-(3.19), (3.10), and (3.3), we arrive at the following result.

Theorem 3.1. For any $u \in \stackrel{\circ}{H}^{1}(\Pi ; \Sigma)^{3}$ we have

$$
\begin{array}{r}
\left\|u ; H^{1}(\Pi(1 / 6))\right\|^{2}+\mathbf{I} X_{h} u ; G_{h} \mathbf{|}^{2}+\left\|u^{\perp} ; H^{1}(B)\right\|+h^{2}\left(H^{2}\left|a^{\prime}\right|^{2}+\left|a_{3}\right|^{2}\right) \\
\leq c_{\Pi}\left\|D\left(\nabla_{x}\right) u ; L_{2}(\Pi)\right\|^{2},
\end{array}
$$

where $X_{h}(Z)=X\left(h^{-1} z\right) ; \mathbf{|} \ldots \mathbf{|}$ is the weighted anisotropic norm (3.3); $u^{\perp}, a_{3}$, and $a^{\prime}=\left(a_{1}, a_{2}, a_{4}, a_{5}, a_{6}\right)^{\top} \in \mathbb{R}^{5}$ are the components in the decomposition (3.11) of the field $u$ on the ball $B$; and $c_{\Pi}$ is a constant independent of $h \in\left(0, h_{0}\right]$.

Let $\mathcal{R}$ be the subspace in $\stackrel{\circ}{H}^{1}(\Pi ; \Sigma)^{3}$ distinguished by the five orthogonality conditions

$$
\int_{B} d^{\prime}\left(y, z-\frac{3}{4}\right)^{\top} u(x) d x=0 \in \mathbb{R}^{6},
$$

where $d^{\prime}(x)$ is the $(3 \times 6)$-matrix obtained from the matrix (3.12) by replacing the unit at the third position in the third column with zero.

Corollary 3.1. For any $u \in \mathcal{R}$ we have

$$
\left\|u ; H^{1}(\Pi)\right\|^{2} \leq C_{\Pi} h^{-2}\left\|D\left(\nabla_{x}\right) u ; L_{2}(\Pi)\right\|^{2},
$$

where $C_{\Pi}$ is a constant independent of $u$ and $h \in\left(0, h_{0}\right]$. 
Proof. It suffices to note that $a^{\prime}=0$ by (3.20), and we have the inequalities

$$
\begin{aligned}
\left\|u ; H^{1}\left(G_{h}\right) \backslash \Pi(1 / 6)\right\|^{2} & \leq c h^{-2} \mid X_{h} u ; G_{h} \mathbf{|}^{2}, \\
\left\|u ; H^{1}(B)\right\|^{2} & \leq c\left(\left\|u^{\perp} ; H^{1}(B)\right\|^{2}+\left|a_{3}\right|^{2}\right),
\end{aligned}
$$

which, together with (3.3), (3.10), (3.7), (3.13), and (3.16), lead to the desired estimate.

3. Existence of a gap in the continuous spectrum. The absolute thresholds (2.51) do not depend on $h>0$. Now we verify that, as this geometric parameter becomes smaller on the interval $\left(\mu_{\dagger}^{0}, 1\right)$, one or several segments of the continuous spectrum of $\mathcal{K}$ separated away from the segment $\left[0, \mu_{\dagger}^{0}\right]$ can be formed.

Proposition 3.1. There exists $h_{0}>0$ such that if $h \in\left(0, h_{0}\right]$ and $\eta \in[0,2 \pi)$, then for the discrete spectrum (2.36) of the operator $m(\eta)$ we have

$$
N(\eta) \geq 6, \quad \Lambda^{(1)}(\eta) \leq \cdots \leq \Lambda^{(5)}(\eta) \leq c_{\Lambda} h^{4}, \quad \Lambda^{(6)}(\eta) \geq C_{\Lambda} h^{2},
$$

with constants $c_{\Lambda}$ and $C_{\Lambda}>0$ independent of $\eta$.

Proof. We employ the max-min principle (see, e.g., [11, Theorem 10.2.2]):

$$
\Lambda^{(j)}(\eta)=\max _{\mathcal{E}_{j}} \inf _{\mathbf{u} \in \mathcal{E}_{j} \backslash\{0\}} \frac{q(\mathbf{u}, \mathbf{u} ; \eta, \Pi)}{\left\|\mathbf{u} ; L_{2}(\Pi)\right\|^{2}},
$$

where $q$ is the Hermitian form (2.6), and $\mathcal{E}_{j}$ is an arbitrary subspace of codimension $j-1$ in $\stackrel{\circ}{H}_{\text {per }}^{1}(\Pi ; \Sigma)^{3}$, i.e., $\operatorname{dim}\left(\stackrel{\circ}{H}_{\text {per }}^{1}(\Pi ; \Sigma)^{3} \ominus \mathcal{E}_{j}\right)=j-1$. We shall construct some special test fields supported on $\bar{B} \cup \bar{G}_{h}$ and, thus, 1-periodic in the variable $y_{1}$. We put

$$
\begin{aligned}
w^{p}(x) & =e_{p} X_{0}(z)-y_{p} e_{3} \partial_{z} X_{0}(z), \\
w^{2+p}(x) & =e_{p}(z-3 / 4) X_{0}(z)-y_{p} e_{3} \partial_{z}\left((z-3 / 4) X_{0}(z)\right), \quad p=1,2, \\
w^{5}(x) & =\left(y_{2} e_{1}-y_{1} e_{2}\right) X_{0}(z), \quad w^{6}(x)=e_{3} X_{0}(z) .
\end{aligned}
$$

Here $e_{p}$ and $e_{3}=(0,0,1)^{\top}$ are the unit vectors of the axes $y_{p}$ and $z$, and $X_{0} \in C^{\infty}(\mathbb{R})$ is a cutoff function equal to 1 for $z>1 / 3$ and to 0 for $z<1 / 6$. Since $X_{0}=1$ on the ball $B$, the restrictions of the fields (3.24) to $B$ are nonzero rigid displacements, so that

$$
\left\|w^{q} ; L_{2}(\Pi)\right\|^{2} \geq c_{0}>0 .
$$

Calculating the strains $\varepsilon_{j k}\left(w^{q}\right)$ (see formulas (1.7)-(1.8)), we find that, among these strains, only the following are nontrivial:

$$
\begin{aligned}
& \varepsilon_{33}\left(w^{p} ; x\right)=-y_{p} \partial_{z}^{2} X_{0}(z), \quad \varepsilon_{33}\left(w^{2+p} ; x\right)=-y_{p} \partial_{z}^{2}\left((z-3 / 4) X_{0}(z)\right), \\
& \varepsilon_{3 p}\left(w^{5} ; x\right)=\varepsilon_{p 3}\left(w^{5} ; x\right)=-(-1)^{p} y_{3-p} \partial_{z} X_{0}(z), \quad \varepsilon_{33}\left(w^{6} ; x\right)=\partial_{z} X_{0}(z) .
\end{aligned}
$$

The derivatives $\partial_{z} X_{0}=\partial X_{0} / \partial z$ are nonzero only for $z \in(1 / 6,1 / 3)$; i.e., the functions (3.26) are supported on the $\operatorname{rod} \overline{G_{h}}$. Clearly, meas $_{3} G_{h}=O\left(h^{2}\right)$ and $\left|y_{p}\right| \leq c h$ for $x \in G_{h}$. Thus,

$$
\begin{aligned}
q\left(w^{q}, w^{q} ; 0, \Pi\right)=\left(A^{0} D\left(\nabla_{x}\right) w^{q}, D\left(\nabla_{x}\right) w^{q}\right)_{\Pi} & \leq c_{q} h^{4}, \quad q=1, \ldots, 5, \\
q\left(w^{6}, w^{6} ; 0, \Pi\right) & \leq c_{6} h^{2} .
\end{aligned}
$$

Now, the inequality $N(\eta) \geq 6$ follows from the next two observations. First, for the vector-valued functions

$$
\mathbf{w}^{q}=\exp \left(-i \eta y_{1}\right) w^{q} \in \stackrel{\circ}{H}_{\mathrm{per}}^{1}(\Pi ; \Sigma)^{3},
$$

the quantities $q\left(\mathbf{w}^{q}, \mathbf{w}^{q} ; \eta, \Pi\right)$ and $\left\|\mathbf{w}^{q} ; L_{2}(\Pi)\right\|^{2}$ coincide with the left-hand sides of inequalities (3.27) and (3.25), respectively. Second, since any subspace $\mathcal{E}_{6}$ of codimension 


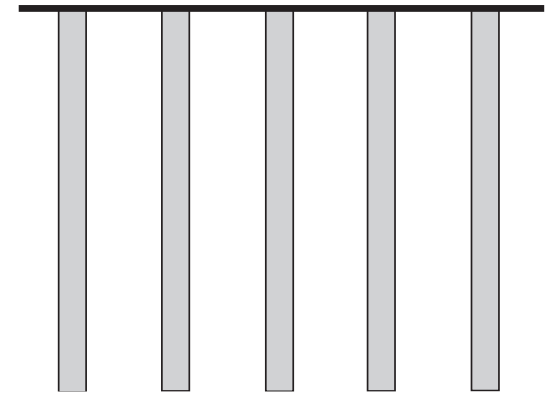

Figure 8. Periodic structure of the clamping zone.

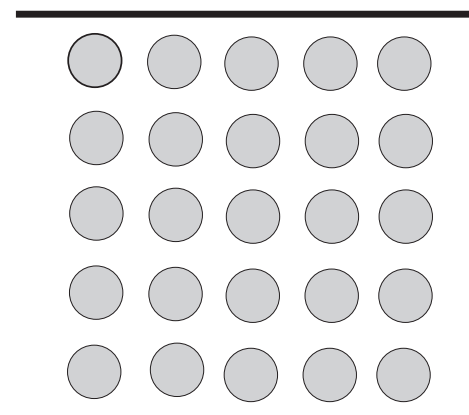

FiguRE 9. Two-periodic structure of the clamping zone.

5 contains a nontrivial linear combination of functions (3.28), it follows that for $j=6$ the right-hand side of (3.23) does not exceed $c h^{2}$ and, if $h$ is small, lies in the halfinterval $\left[0, \Lambda_{\min }(\eta)\right)$. Therefore, by [11, Theorem 10.2.2], the operator $m(\eta)$ has at least 6 eigenvalues on that half-interval.

The same theorem and formulas (3.26) and (3.25) show that $\Lambda^{(5)}(\eta) \leq c h^{4}$. It remains to verify the last relation on the list (3.22). For the role of $\mathcal{E}_{6}$ we take the subspace $\mathcal{R}(\eta)$ of vector-valued functions $\mathbf{u}=\exp \left(-i \eta y_{1}\right) u$ with $u \in \mathcal{R}$. The first observation made above and inequalities (1.9) and (3.21) imply that

$$
\left\|\mathbf{u} ; L_{2}(\Pi)\right\|^{-2} q(\mathbf{u}, \mathbf{u} ; \eta, \Pi) \geq c_{A}\left\|u ; L_{2}(\Pi)\right\|^{-2}\left\|D\left(\nabla_{x}\right) u ; L_{2}(\Pi)\right\|^{2} \geq c_{A} C_{\Pi}^{-1} h^{2},
$$

whence $\Lambda^{(6)}(\eta) \geq c_{A} C_{\Pi}^{-1} h^{2}$.

Thus, the segments $\Delta^{(1)}, \ldots, \Delta^{(5)}$ of the continuous spectrum of $\mathcal{K}$ (see (2.52) ) lie in the set $\left[\left(1+c_{\Lambda} h^{4}\right)^{-1}, 1\right]$, and the segments $\Delta^{(n)}$ with $n \geq 6$ lie in $\left[0,\left(1+C_{\Lambda} h^{2}\right)^{-1}\right]$. If $h$ is small, then these sets do not intersect; therefore, the continuous spectrum of $\mathcal{K}$ necessarily has at least one gap.

4. Generalizations and consequences. The Dirichlet boundary conditions (1.6) are necessary for building an example of a waveguide with a gap in the continuous spectrum. Such conditions may be posed on one of the faces only, or even on a union of stripes in that face (Figure 8). However, in the case of the Neumann conditions (1.5) on the entire boundary $\partial \Omega=: \Sigma$, the absolute threshold $\lambda_{\dagger}^{0}$ (see (2.50) becomes zero, because for $\eta=\zeta=0$ the model problem (2.9) on the section $\Theta$ acquires the zero eigenvalue. As a result, all of the half-axis $\overline{\mathbb{R}_{+}}$(all of the segment $[0,1]$ ) becomes filled with the essential spectrum of problem (1.4)-(1.6) (of the operator $\mathcal{K}$ ). 


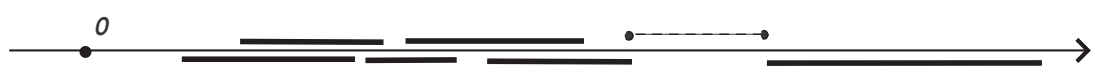

Figure 10. A gap between the fifth and sixth segments.

It may be assumed that, under the stabilization conditions (1.10), the rigidity matrix $A^{0}$ and the density $\rho_{0}$ of the material depend $l$-periodically on the variable $x_{3}=z$. In that case, instead of the Fourier transformation (2.8) we should apply the Gelfand transformation (2.2) relative to the variable $l^{-1} z$, which leads to a model problem on the parallelepiped $\Theta \times(0,1)$ with periodicity conditions on its upper and lower faces. The same modifications are required if the Dirichlet conditions are posed on a set periodic in the two directions $x_{1}$ and $z$ (Figure 9).

The form of the joints (3.6) is not of principal importance. What is important indeed is that two massive bodies are connected with a thin and long cushion (Figures 3 and 4). We also remind the reader about the Dirichlet conditions on the lower prism. The construction is low-sensitive to the elastic properties of the material: all bounds in the estimates of Subsection 3 in $\S 3$ are determined by the constants $c_{A}^{0}, C_{A}^{0}$ and $c_{\rho}^{0}, C_{\rho}^{0}$ occurring in (1.9), but not by the rigidity matrix $A^{0}$ and the density $\rho_{0}$ themselves.

The gap discovered is depicted in Figure 10. It is located between the first five and the sixth segment of the essential spectrum and is marked by a dashed line. The author does not know whether there are gaps to the right or to the left of that one.

If a single ball in the half-layer given by formulas (1.3) and (3.6) is soft and/or heavy compared to the other part of the elastic joint, then, by using the max-min principle (3.23) with test functions localized in that ball, it is not difficult to verify (cf. [47]) the following statement: if the characteristics of the material are chosen appropriately, then an arbitrarily small interval $(0, \delta)$ of the continuous spectrum of problem (1.4)-(1.6) can contain any preassigned number of eigenvalues. No example of an elastic waveguide for which the gap contains an eigenvalue is known.

\section{REFERENCES}

[1] Rayleigh, Lord, On waves propagating along the plane surface of an elastic solid, Proc. London Math. Soc. 17 (1885), 4-11.

[2] A. S. Bonnet-Bendhia, J. Duterte, and P. Joly, Mathematical analysis of elastic surface waves in topographic waveguides, Math. Models Methods Appl. Sci. 9 (1999), no. 5, 755-798. MR1697393 (2000d:74036)

[3] I. V. Kamotskiu and A. P. Kiselev, Energy approach to the proof of the existence of Rayleigh waves in an anisotropic halfspace, Prikl. Mat. Mekh. 73 (2009), no. 4, 645-654. (Russian)

[4] S. A. Nazarov, Rayleigh waves for an elastic half-layer with a partially damped periodic boundary, Dokl. Akad. Nauk 423 (2008), no. 1, 56-61. (Russian) MR2494246

[5] S. G. Lekhnitskiǔ, Theory of elasticity of an anisotropic body, Nauka, Moscow, 1977; English transl., Mir, Moscow, 1981. MR0502604 (58:19575) MR0610607 (82d:73001)

[6] S. A. Nazarov, Asymptotic theory of thin plates and rods. Dimension reduction and integral estimates. Vol. I, Nauchn. Kniga, Novosibirsk, 2002. (Russian)

[7] F. Ursell, Mathematical aspects of trapping modes in the theory of surface waves, J. Fluid Mech. 183 (1987), 421-437. MR0919481 (89a:76019)

[8] C. M. Linton and P. McIver, Embedded trapped modes in water waves and acoustics, Wave Motion 45 (2007), 16-29. MR2441664 (2009e:35216)

[9] O. A. Ladyzhenskaya, Boundary value problems of mathematical physics, Nauka, Moscow, 1973; English transl., Appl. Math. Sci., vol. 49, Springer-Verlag, New York, 1985. MR0599579 (58:29032) MR.0793735 (87f:35001)

[10] J.-L. Lions and E. Magenes, Problèmes aux limites non homogènes et applications. Vol. 1, Travaux et Recherches Math., No. 17, Dunod, Paris, 1968. MR0247243(40:512) 
[11] M. Sh. Birman and M. Z. Solomyak, Spectral theory of selfadjoint operators in Hilbert space, Leningrad. Univ., Leningrad, 1980; English transl., Math. Appl. (Soviet Ser.), D. Reidel Publ. Co., Dordrecht, 1987. MR0609148 (82k:47001) MR1192782 (93g:47001)

[12] V. A. Kondrat'ev, Boundary value problems for elliptic equations in domains with conical or angular points, Trudy Moskov. Mat. Obshch. 16 (1967), 209-292; English transl. in Trans. Moscow Math. Soc. 1967 (1968). MR0226187 (37:1777)

[13] V. G. Maz'ya and B. A. Plamenevskiॅ, The coefficients in the asymptotics of solutions of elliptic boundary value problems with conical points, Math. Nachr. 76 (1977), 29-60. (Russian) MR0601608 $(58: 29176)$

[14] Estimates in $L_{p}$ and in Hölder classes, and the Miranda-Agmon maximum principle for the solutions of elliptic boundary value problems in domains with singular points on the boundary, Math. Nachr. 81 (1978), 25-82. (Russian) MR0492821 (58:11886)

[15] S. A. Nazarov, Polynomial property of selfadjoint elliptic boundary value problems, and the algebraic description of their attributes, Uspekhi Mat. Nauk 54 (1999), no. 5, 77-142; English transl., Russian Math. Surveys 54 (1999), no. 5, 947-1014. MR 1741662 (2001k:35073)

[16] S. A. Nazarov and B. A. Plamenevskiŭ, Elliptic problems in domains with piecewise smooth boundary, Nauka, Moscow, 1991; English variant, de Gruyter Exp. Math., vol. 13, Walter de Gruyter and Co., Berlin, 1994. MR.1283387 (95h:35001)

[17] V. A. Kozlov, V. G. Maz'ya, and J. Rossmann, Elliptic boundary value problems in domains with point singularities, Math. Surveys Monogr., vol. 52, Amer. Math. Soc., Providence, RI, 1997. MR1469972 (98f:35038)

[18] S. A. Nazarov, Asymptotic behavior of solutions of a problem in elasticity theory for a threedimensional body with thin extensions, Dokl. Akad. Nauk 352 (1997), no. 4, 458-461. (Russian) MR:1450987 (99f:73032)

[19] Korn's inequalities for junctions of spatial bodies and thin rods, Math. Methods Appl. Sci. 20 (1997), no. 3, 219-243. MR1430494 (98b:73009)

[20] V. A. Kozlov, V. G. Maz'ya, and A. B. Movchan, Asymptotic analysis of fields in multi-structures, Clarendon Press, Oxford Univ. Press, New York, 1999. MR1860617 (2002j:35003)

[21] S. A. Nazarov, Asymptotic analysis and modeling of the junction of a massive body and thin rods. Trudy Sem. I. G. Petrovsk. No. 24 (2004), 95-214; English transl., J. Math. Sci. (N. Y.) 127 (2005), no. 5, 2192-2262. MR2360841 (2009a:74087)

[22] P. A. Kuchment, Floquet theory for partial differential equations, Uspekhi Mat. Nauk 37 (1982), no. 4, 3-52; English transl., Russian Math. Surveys 37 (1982), no. 4, 60-94. MR0667973 (84b:35018)

[23] A. Figotin and P. Kuchment, Band-gap structure of spectra of periodic dielectric and acoustic media. I. Scalar model, SIAM J. Appl. Math. 56 (1996), 68-88; II. Two-dimensional photonic crystals, ibid. 56 (1996), 1561-1620. MR.1372891 (97g:35122) MR1417473 (98f:35012)

[24] M. M. Skriganov, Geometric and arithmetic methods in the spectral theory of multidimensional periodic operators, Trudy Mat. Inst. Steklov. 171 (1985), 122 pp.; English transl., Proc. Steklov Inst. Math. 1987, no. 2 (171). MR0798454 (87h:47110), MR0905202 (88g:47038)

[25] P. Kuchment, Floquet theory for partial differential equations, Oper. Theory Adv. Appl., vol. 60, Birkhäuser, Basel, 1993. MR1232660 (94h:35002)

[26] E. L. Green, Spectral theory of Laplace-Beltrami operators with periodic metrics, J. Differential Equations 133 (1997), 15-29. MR:1426755 (97k:58168)

[27] R. Hempel and K. Lineau, Spectral properties of periodic media in the large coupling limit, Comm. Partial Differential Equations 25 (2000), 1445-1470. MR1765136 (2001h:47074)

[28] L. Friedlander, On the density of states of periodic media in the large coupling limit, Comm. Partial Differential Equations 27 (2002), 355-380. MR1886963 (2003d:35194)

[29] V. V. Zhikov, On spectrum gaps of some divergent elliptic operators with periodic coefficients, Algebra i Analiz 16 (2004), no. 5, 34-58; English transl., St. Petersburg Math. J. 16 (2005), no. 5, 773-790. MR.2106666 (2006a:35053)

[30] N. Filonov, Gaps in the spectrum of the Maxwell operator with periodic coefficients, Comm. Math. Phys. 240 (2003), no. 1-2, 161-170. MR2004984 (2004i:35293)

[31] P. Kuchment, The mathematics of photonic crystals, Mathematical Modeling in Optical Science, Frontiers Appl. Math., vol. 22, SIAM, Philadelphia, PA, 2001, pp. 207-272. MR1831334 (2002k:78002)

[32] I. M. Gel'fand, Expansion in characteristic functions of an equation with periodic coefficients, Dokl. Akad. Nauk SSSR 73 (1950), 1117-1120. (Russian) MR0039154 (12:503a)

[33] S. A. Nazarov, Elliptic boundary value problems with periodic coefficients in a cylinder, Izv. Akad. Nauk SSSR Ser. Mat. 45 (1981), no. 1, 101-112; English transl. in Math. USSR-Izv. 18 (1982), no. 1. MR0607578 (82e:35035) 
[34] I. Ts. Gokhberg and M. G. Kreŭn, Introduction to the theory of linear nonselfadjoint operators in Hilbert space, Nauka, Moscow, 1965; English transl., Transl. Math. Monogr., vol. 18, Amer. Math. Soc., Providence, RI, 1969. MR0220070 (36:3137) MR0246142 (39:7447)

[35] M. S. Agranovich and M. I. Vishik, Elliptic problems with a parameter and parabolic problems of general type, Uspekhi Mat. Nauk 19 (1964), no. 3, 53-161; English transl. in Russian Math. Surveys 19 (1964), no. 3. MR0192188(33:415)

[36] J. Nečas, Les méthodes directes en théorie des équations elliptiques, Masson, Paris, 1967. MR0227584 (37:3168)

[37] V. A. Kondrat'ev and O. A. Oleınnik, Boundary value problems for a system in elasticity theory in unbounded domains. Korn inequalities, Uspekhi Mat. Nauk 43 (1988), no. 5, 55-98; English transl., Russian Math. Surveys 43 (1988), no. 5, 65-119. MR0971465 (89m:35061)

[38] T. Kato, Perturbation theory for linear operators, 2nd ed., Grundlehren Math. Wiss., Bd. 132, Springer-Verlag, Berlin-New York, 1976. MR0407617 (53:11389)

[39] K. O. Friedrichs, On the boundary-value problems of the theory of elasticity and Korn's inequality, Ann. of Math. (2) 48 (1947), 441-471. MR0022750 (9:255b)

[40] P. P. Mosolov and V. P. Myasnikov, A proof of Korn's inequality, Dokl. Akad. Nauk SSSR 201 (1971), no. 1, 36-39; English transl., Soviet Math. Dokl. 12 (1971), 1618-1622. MR0296685 $(45: 5744)$

[41] G. Duvaut and J.-L. Lions, Inequalities in mechanics and physics, Grundlehren Math. Wiss., Bd. 219, Springer-Verlag, Berlin-New York, 1976. MR0521262 (58:25191)

[42] B. A. Shoukhet, On asymptotically exact equations of thin plates of complex structures, Prikl. Mat. Mekh. 37 (1973), no. 5, 914-924; English transl., J. Appl. Math. Mech. 37 (1973), 867-877 (1974). MR0349110 (50:1604)

[43] S. A. Nazarov, Korn inequalities that are asymptotically exact for thin domains, Vestnik S.Peterburg. Univ. Mat. Mekh. Astronom. 1992, vyp. 2, 19-24; English transl., Vestnik St. Petersburg Univ. Math. 25 (1992), no. 2, 18-22. MR.1280920 (95m:35026)

[44] _ Korn's inequalities for elastic joints of massive bodies, thin plates, and rods, Uspekhi Mat. Nauk 63 (2008), no. 1, 37-110; English transl., Russian Math. Surveys 63 (2008), no. 1, 35-107. MR 2406182(2009c:35457)

[45] Justification of the asymptotic theory of thin rods. Integral and pointwise estimates, Probl. Mat. Anal., Vyp. 17, S.-Peterburg. Gos. Univ., St. Petersburg, 1997, pp. 101-152; English transl., J. Math. Sci. (N. Y.) 97 (1999), no. 4, 4245-4279. MR1788230 (2002d:74045)

[46] _ Korn inequality for elastic junction of a body with a rod, Problems of Mechanics of Deformed Hard Body, S.-Peterburg. Gos. Univ., St. Petersburg, 2002, pp. 234-240. (Russian)

[47] - Trapped modes for a cylindrical elastic waveguide with a damping gasket, Zh. Vychisl. Mat. Mat. Fiz. 48 (2008), no. 5, 863-881; English transl. in Comput. Math. Math. Phys. 48 (2008), no. 5. MR2433645 (2009e:74061)

Institute of Engineering Problems, Russian Academy of Sciences, 61 Bol'shoi Prospekt V.O., St. Petersburg 199178, Russia

E-mail address: serna@snark.ipme.ru, srgnazarov@yahoo.co.uk

Received 8/MAY/2008

Translated by A. PLOTKIN 